"Macromolecular design of semiconductive polymers towards thermodynamically stable organic photovoltaic active materials and devices"

\section{Georges HADZIIOANNOU}

Institut Universitaire de France

Chaire Arkema/Région Aquitaine

Laboratoire de Chimie des Polymères Organiques (LCPO) - UMR5629

Université Bordeaux 1 / Institut Polytechnique de Bordeaux (ENSCBP) / CNRS

\section{Email :hadzii@enscbp.fr}

The growing interest for renewable energy technologies, such as photovoltaic (PV) devices, combined with the need for low-cost processing, have contributed to the quick expansion of organic photovoltaics. Considerable efforts have focused on the development of donor/acceptor (D/A) heterojunctions based on photoactive compounds of electron-donating conjugated polymers and fullerene derivatives. In these devices, the organic components form, throughout the entire active layer, nanometer-sized D and A domains at whose interfaces photogenerated excitons can dissociate into free charge carriers, which in turn are driven to the collecting electrodes by the device build-in electric field. A key issue involves the precise control of film morphology, down to nanometer dimensions. The use of blockcopolymers provides an opportunity to use self-assembly and achieve control over morphology. In the present talk, we report on the thin film structure of polymer/fullerene blends nanostructured and thermodynamically stabilized with block copolymers and on the optoelectronic properties of solar cells made thereof.

Acknowledgements. The following people are gratefully acknowledged for their contribution Henri Cramail, Eric Cloutet, Cyril Brochon, Guillaume Fleury, Lionel Hirsh, Laurence Vignau, Guillaume Wantz and Raffaele Mezzenga.

Polymers \& Colloids, Lecture

Carbon-rich nanostructures from molecular precursors

Tobias Hoheisel $^{1}$, Stephen Schrettl ${ }^{2}$, Ruth Szilluweit ${ }^{2}$, Holger Frauenrath $^{2}$

${ }^{1}$ ETH Zürich, Department of Materials, Wolfgang-Pauli-Str. 10, 8093 Zürich

${ }^{2}$ École Polytechnique Fédérale de Lausanne, EPFL-STI-IMX-LMOM, Station 12, 1015 Lausanne

Carbonaceous materials with a feature-size on the nanometer-scale offer considerable prospects for emerging technologies such as lithium batteries or hydrogen storage. Currently, the methods most widely employed to prepare carbon nanostructures rely on harsh reaction conditions, which limit a functionalization to post-synthetic steps. Our strategy for the preparation of nanostructured carbonaceous material is based on the synthesis of amphiphilic oligo(ethynylene)s. We recently reported a protocol for the convenient synthesis of acetylated glycosylated oligo(ethynylene)s.[1] After deprotection, the molecules resemble typical glycolipids. In polar protic solutions, these molecules self-assemble into colloidal aggregates which can be carbonized by a soft external stimulus such as UV light. Exploiting this strategy, the preparation of carbon-rich nanostructures with a controlled morphology and a defined surface modification can be envisaged.

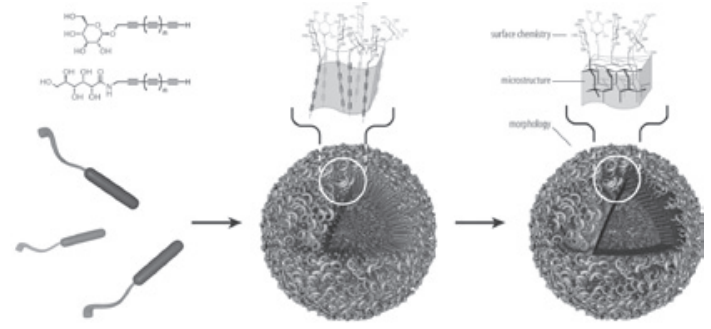

[1] Hoheisel, T. N.; Frauenrath, H. Organic Letters 2008, 10, 4525.

\section{Access to Controlled Self-Assembly from Fibers to Micelles of a Lysine} Rich Amphiphilic Peptide via Point Mutations

\author{
Thomas Schuster, Dirk de Bruyn Ouboter, Wolfgang P. Meier
}

University Basel, Klingelbergstrasse 80,4055 Basel, Switzerland

Self-assembled nanostructures and functional smart materials from blockco-polymers, peptide based hybrid or pure peptide material attract increasing interest e.g. for potential application as drug delivery systems. A small uncharged amphiphilic peptide of only ten amino acids was found to form massive microspheres ${ }^{1}$. Similar to this we synthesised a series of charged amphiphilic compounds, in total ten oligopeptides containing three parts: (a) a charged lysine part (b) an acetylated lysine part and (c) a hydrophobic helix. Latter was inspired by gramicidin A and possesses a defined secondary structure $^{2}$. By stepwise exchange of single amino acids from acetylated lysine into lysine the effect of point mutation of the primary sequence on the final assembly morphology could be investigated. Thereby the overall amphiphilicity was controlled over the ratio of $a$ to $b$ of the hydrophilic parts with a constant c value. Circular Dichroism (CD), dynamic light scattering (DLS), surface tension and transmission electron microscopy (TEM) served as analytical tools for the assembled structures. We observed a change in secondary structure which comes along with a change in the morphology of the assembly from balls/fibers to micelles. The charge library is divided in three different regimes of similar behaviour: At high acetylation degree we found a change in secondary structure of the hydrophobic part into $\beta$-sheet like structure and fiber formation, moderate acetylation gave importance to the hydrophobic part and at low acetylation degree the hydrophilic part defined the properties.

[1] C. Dittrich, D. DeBruynOuboter, T. Schuster and W. Meier, Biomaterials, 2010, submitted.

[2] B. A. Wallace, Journal of Structural Biology, 1998, 121, 123-141.

Polymers \& Colloids, Lecture

\section{Polymer/Peptide Therapeutics Against the Entry of HIV Type 1}

Maarten Danial $^{1}$, Swetha Uppalapati ${ }^{2}$, Andy J. G. Pötgens ${ }^{3}$, Michael J. Root $^{2}$, Harm-Anton Klok ${ }^{1}$

${ }^{1}$ École Polytechnique Fédérale de Lausanne, Institut des Matériaux, Bâtiment MXD, Station 12, 1015 Lausanne, Switzerland, ${ }^{2}$ Thomas Jefferson University, Department of Biochemistry and Molecular Biology, Philadelphia, Pennsylvania 19107, United States of America, and ${ }^{3}$ AplaGen GmbH, D-52499 Baesweiler, Germany.

The acquired immune deficiency syndrome (AIDS), caused by the human immunodeficiency virus (HIV), is one of the world's deadliest diseases known today [1]. Although several therapeutics have been developed to enable to reduce the viral loads and improve patients quality of life, no known cure is available. Additionally, the vast majority of the current therapeutics are inhibitors to enzymes of HIV type 1 (HIV-1) e.g. reverse transcriptase, integrases, that are active in the infected cell [2]. Alternatively, we have explored the world of synthetic polymers and combined this with naturally occurring polymers, particularly peptides, which target and bind to HIV-1 prior to host cell membrane penetration. We will present an overview of the design as well as the synthetic, biophysical and biological characteristics of various novel peptides and polymer-peptide conjugate architectures, which have the ultimate purpose of reducing HIV-1 entry into host cells.

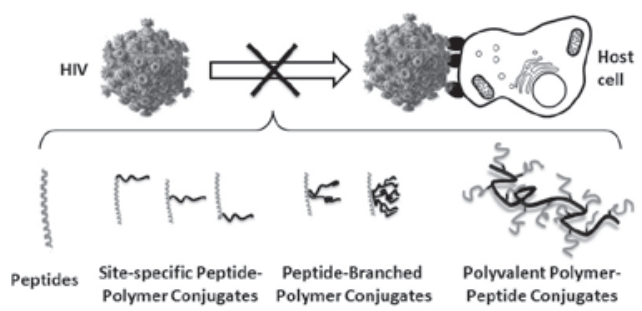

[1] World Health Organization Fact Sheet 310, Nov. 2008.

[2] Mehellou, Y., De Clercq, E. J. Med. Chem. 2010, 53, 521. 
Poly(diarylstannane)s: $\sigma-\pi$ conjugated organometallic polymers

\section{Markus Trummer, Paul Smith, Walter Caseri}

Department of Materials, Eidgenössische Technische Hochschule (ETH) Zurich, Wolfang-Pauli-Strasse 10, CH-8093 Zurich, Switzerland

Organometallic polymers with metal atoms in the polymer backbone are a unique class of materials with exceptional materials properties. So far the most promising method to prepare polystannanes was dehydropolymerzation of dialkylstannanes, $\mathrm{R}_{2} \mathrm{SnH}_{2}(\mathrm{R}=$ alky group), with Wilkinson's catalyst, $\left[\mathrm{RhCl}\left(\mathrm{PPh}_{3}\right)_{3}\right]^{[1]}$. Unfortunately analogous reactions did not proceed with diarylstannanes, $\mathrm{Ar}_{2} \mathrm{SnH}_{2}$. Therefore we adopted the reaction of dichlorodiorganostannanes with sodium in liquid ammonia ${ }^{[2,3]}$ to obtain the desired polymers. The course of the polymerization was followed by electrical conductivity measurements and in-situ ${ }^{119} \mathrm{Sn}-\mathrm{NMR}$ spectroscopy in liquid ammonia. We demonstrated that this reaction does not follow the proposed route via the intermediate organotin dianion, but two intermediates are formed, namely $\left(\mathrm{Ph}_{2} \mathrm{Sn}-\mathrm{SnPh}_{2}\right)^{2-}$ and $\mathrm{Ph}_{2} \mathrm{SnH}^{-}$. With this knowledge it was possible to adapt the reaction conditions to obtain pure poly(diphenylstannane) and other aryl derivatives to diversify the materials properties. While poly(dialkylstannane)s exhibit $\sigma$-delocalization of the electrons along the polymer backbone and, therefore, feature an absorption maximum at around $400 \mathrm{~nm}$ in UV/Vis spectra, a significant red shift was achieved by replacing the alkyl side chains with aryl groups due to the $\sigma-\pi$ delocalization along the polymer backbone and the aromatic groups.

[1] F. Choffat, D. Schmid, W. Caseri, P. Wolfer and P. Smith, Macromolecules 2007, 40, 7878-7889.

[2] R. F. Chambers and P. C. Scherer, J. Am. Chem. Soc. 1926, 48, 1054 1062.

[3] C. A. Kraus and W. N. Greer, J. Am. Chem. Soc. 1925, 47, 2568-2575.
Polymers \& Colloids, Lecture

528

Systematic coarse graining flowing polymer melts: thermodynamically guided simulations and resulting constitutive models

Patrick Ilg, Hans Christian Öttinger, Martin Kröger

Polymer Physics, ETH Zürich, Wolfgang-Pauli-Str. 10, CH-8093 Zürich

The derivation of reliable constitutive equations for flowing complex fluids and the prediction of macroscopic behavior from their microstructures still presents major challenges to date [1]. Here, we present a systematic coarsegraining method for microscopic models of complex fluids that is able to efficiently bridge the time- and length scale gap between the microscopic level and macroscopic dynamics. The systematic coarse-graining method is exemplified for low-molecular polymeric systems subjected to homogeneous flow an alternating Monte-Carlo-molecular dynamics iteration scheme in order to obtain the model equations for the slow variables [2]. For chosen flow situations of interest, the established model predicts structural as well as material functions beyond the regime of linear response. The results are in quantitative agreement with those obtained via standard nonequilibrium molecular

Furthermore, we take several steps towards establishing the functional form of the macroscopic constitutive model by reconstructing the thermodynamic building blocks from the alternating Monte-Carlo-molecular dynamics simulations. For low-molecular polymer melts, the constitutive model we propose shows shear thinning, stress overshoots, normal stress coefficients and elongational viscosities in agreement with reference results. The constitutive equation can be interpreted as a molecularly derived Giesekus model with configuration- and rate-dependent coefficients.

[1] P. Ilg, V.G. Mavrantzas, H.C. Öttinger, Multiscale Modeling and Coarse Equilibrium Thermodynamics, in: Modeling and Simulations in Polymers, P.D. Gujrati and A.L. Leonov (Eds.). (Wiley-VCH, 2010).

[2] P. Ilg, H.C. Öttinger, M. Kröger, Phys. Rev. E 2009, 79, 011802. fields. We use established concepts of nonequilibrium thermodynamics and dynamics simulations. Graining of Polymer Dynamics: Simulations Guided by Statistical Beyond-
Preparation of catalytically active, covalent enzyme-polymer conjugates

$$
\text { Andrea Grotzky, Peter Walde, A. Dieter Schlüter }
$$

Polymer Chemistry, Department of Materials, ETH Zürich, WolfgangPauli-Strasse 10, CH-8093 Zürich, Switzerland

Coupling peptides/proteins to synthetic polymers is a highly interesting and potential applications including drug delivery. ${ }^{3}$ Special cases are enzymepolymer conjugates. We present the biofunctionalization of a polymer chain with a number of enzymes.

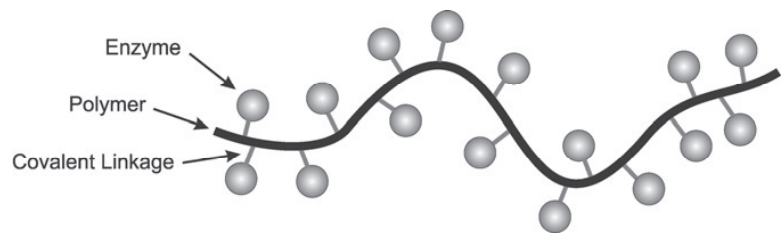

For covalent linkage between a polymer and an enzyme hydrazone bond formation was investigated. Model studies with $\alpha$-poly-D-lysine as polymer and horseradish peroxidase as enzyme showed promising results. The kinetics of the bis-aryl hydrazone bond formation was followed by UV/vis spectroscopy and reversed phase HPLC. Preservation of the tertiary structure and of the activity of the enzyme were confirmed by circular dichroism and activity measurements. The use of other types of synthetic polymers is currently under investigation.

[1] Gauthier, M. A., Klok, H. A., Chem. Comm. 2008, 23, 2591

[2] Lutz, J. F., Börner, H. G., Prog. Polym. Sci. 2008, 33, 1.

[3] Duncan, R., Nat. Rev. Cancer 2006, 6, 688. ongoing research topic. ${ }^{1,2}$ Protein-polymer conjugates have a wide range of

Aspects of the chemistry of polyalkylene glycols in providing energy efficient and environmental solutions for lubricants of the future

\author{
Dr Martin Greaves \\ The Dow Chemical Company \\ Bachtobelstrasse 3 \\ Horgen, CH-8810, Switzerland
}

\section{ABSTRACT}

Lubricants are a vital component of today's society. Without lubricants there would be no transportation industry and no manufacturing industry to drive our economies. Most lubricants today are designed from natural hydrocarbon base oils that are sourced from oil refining. But our planet may only have a few decades of natural oil in reserve. The chemical industry is playing an important role in developing new synthetic lubricants to provide energy efficient solutions by improving friction control in equipment thereby minimizing fuel and energy consumption to power equipment.

Polyalkylene glycols (PAG) will be an important family of polymers in the design of future lubricants. Friction characteristics are dependent on the polymer architecture and choice of alkylene oxides that are used in their synthesis. Furthermore, if mixed oxides are used, random, block and reverse block structures can be formed which can impart different lubrication behaviour. Aspects of friction behaviour versus polymer architecture will be discussed and how this impacts the design of wind turbine lubricants, hydraulic fluids and automotive engine oils where energy conservation through better friction control is ever more critical.

Polyalkylene glycols can also play an important role in designing environmentally acceptable lubricant solutions as alternatives to petroleum oils which are not biodegradable and form sheens when leaks and spills occur into waterways. By carefully engineering PAGs, it is possible to design lubricants that are water soluble, non-sheening and highly biodegradable and provide a practical solution to improving the environmental sustainability of lubricants. 


\section{Impact of the chemical structure on the polymerization of cationic} monomers and solution behavior of the resulting polyelectrolytes

\author{
Hamideh Ahmadloo, Ricardo Losada, Christine Wandrey
}

EPFL, Institut de Bioingénierie, Laboratoire de Médecine Régénérative et de Pharmacobiologie, station 15, 1015 Lausanne, Switzerland

Water-soluble cationic polymers, cationic polyelectrolytes, are increasingly interesting due to their widespread already established or potential application fields. Primarily electrostatic effects present during the polymerization process cause deviations of the polymerization kinetics from the ideal case However, also other than electrostatic interactions cannot be neglected. Despite practical importance, appropriate kinetic-mechanistic studies are rare.

The availability of the two double-charged cationic monomers 1,3-bis (N,N,N-trimethylammonium)-2-propylmethacrylate dichloride (di-M) and 1,3-bis(N,N,N-trimethylammonium)-2-propylacrylate dichloride (di-A) allowed for studying the free radical polymerization under strong electrostatic influences and quantifying this impact comparing the kinetic results with those of the mono-charged analogue N,N,N-trimethylammonium-2propylacrylate chloride (Q9). The monomer exponents of the overall polymerization rate equation were obtained as $4.4,3.5$, and 2.0 for di-M, di-A and Q9, respectively. Both the charge density and the chemical structure of the polymer backbone unit were identified to influence the reaction kinetics.

It was also demonstrated that both the charge density and the chemical structure of the polymer backbone govern the solution behavior of the resulting polyelectrolytes, the hydrolytic stability in aqueous solution, and the complex formation characteristics with oppositely charged polyelectrolytes.

Acknowledgement: The research is supported by the SNSF, grants 200021107737/1 and 200020_119818/1

[1] R. Losada, C. Wandrey, Macromol. Rapid Commun. 2008, 29, 252

[2] R. Losada, C. Wandrey, Macromolecules 2009, 42, 3285.

[3] R. Losada, H. Ahmadlooh, C. Wandrey, CHIMIA, 2010, 64, in press.

Polymers \& Colloids, Invited Lecture

\section{Dynamics of flowing colloidal suspensions}

\section{Matthias Ballauff}

Soft Matter and Functional Materials, Helmholtz-Zentrum Berlin, and Institute of Physics, Humboldt-Universität zu Berlin, Germany

We present our recent experiments on the dynamics of colloidal particles and the comparison with mode-coupling theory (MCT). The colloidal particles used in our experiments consist of a solid core onto which a thermosensitive network of poly(N-isopropylacrylamide) has been grafted. Immersed in water these particles will swell at room temperature. Raising the temperature then leads to a de-swelling of the particles. In this way the effective volume fraction can be adjusted in a simple and very precise way.

In this contribution, we shall discuss the following topics:

Measurements in continuous flow: Here we discuss in particular measurements of the linear-viscoelastic behaviour of our thermosensitive suspension. The flow curves of the suspension can be compared to $G^{\prime}$ and $G$ ', measured over nine decades in frequency [1]. MCT is shown to provide an excellent descript of the fluid-to-glass transition in these suspensions.

Measurements in discontinuous flow: This last section is devoted to recen measurements in non-stationary flow. Here we present work done with e.g. Fourier-rheology on our suspensions. MCT is shown to predict the nonstationary flow based on parameters derived from stationary experiments.

[1] Winter, HH; Siebenburger, M; Hajnal, D; Henrich, O; Fuchs, M; Ballauff, M, An empirical constitutive law for concentrated colloidal suspensions in the approach of the glass transition, Rheol. Acta 2009, 48, 747753

\section{Stability of Ketoprofen-PLGA Nanocomposites produced by Supercritical Fluid Extraction of Emulsions}

\author{
Johannes Kluge ${ }^{1}$, Gerhard Muhrer ${ }^{2}$ and Marco Mazzotti ${ }^{1}$
}

${ }^{1}$ ETH Zurich, Institute of Process Engineering, Sonneggstr. 3, 8092 Zurich

${ }^{2}$ Novartis Pharma AG, Lichtstrasse 35, 4056 Basel, Switzerland

Poly(lactic-co-glycolic)acid, PLGA, is a biodegradable and biocompatible polymer, representing a well-investigated delivery system for many active pharmaceutical ingredients (API's). Micro-encapsulation of API's into polymer particles is often achieved using an emulsion process, and in this context, supercritical $\mathrm{CO}_{2}$ may be used as solvent extracting agent. Supercritical Fluid Extraction of Emulsions (SFEE) combines the convenience of an emulsion process, i.e. tight control of particle size, with the advantages of a $\mathrm{scCO}_{2}$ process, i.e. high product purity and mild operating conditions [1].

SFEE may be used to encapsulate poorly water-soluble API's into amorphous PLGA-based micro- and nanoparticles [2]. Such nanocomposites are interesting for innovative drug delivery concepts such as controlled release or drug targeting. For a sufficient product shelf-life, long-term stable drugpolymer co-formulations are required. However, there is a lack of methods and also of concepts to investigate fundamentally the stability of drugpolymer composites and the underlying compatibility of API and polymer.

In this work, the equilibration of PLGA with crystalline ketoprofen (KET), a chiral anti-inflammatory drug, is studied using colloidal PLGA obtained by SFEE [2]. The solubility of KET in PLGA nanoparticles is determined, and the equilibrium of KET and PLGA is described using a suitable model. The present findings allow detailed conclusions concerning the stability of KETPLGA composites, and enable the design of delivery systems combining a maximized drug load with an extended shelf-life.

[1] J. Kluge, F. Fusaro, N. Casas, G. Muhrer and M. Mazzotti, J. Supercrit. Fluids 2009, 50, 327-335.

[2] J. Kluge, F. Fusaro, G. Muhrer and M. Mazzotti, J. Supercrit. Fluids 2009, 50, 336-343.

Polymers \& Colloids, Lecture

Depletion interactions in charged, aqueous colloid/polymer-mixtures

Kitty van Gruijthuijsen ${ }^{1}$, Marc Obiols-Rabasa ${ }^{1}$, Remco Tuinier ${ }^{2}$, Peter Schurtenberger ${ }^{1}$, Anna Stradner

${ }^{1}$ Adolphe Merkle Institute, University of Fribourg, Rte de l'Ancienne Papeterie, CH-1723 Marly, Switzerland

${ }^{2}$ DSM Research, ACES, P.O. Box 18, 6160 MD Geleen, The Netherlands

Food and materials sciences have started to enormously profit from parallel developments in soft matter physics. Especially insight into model systems without specific interactions, like hard spheres, charged spheres, and ideal polymers, can easily be extrapolated to their industrial counterparts. In mixtures of these model components, steric exclusion of the polymer from an area around the colloid results in an attractive colloid-colloid interaction potential, the so-called depletion attraction. Theoretical models to predict depletion-induced phase behaviour apply both to organic model systems, as well as to mixtures of natural ingredients [1]. In a next step towards reality's complexity we introduce electrostatic repulsions between the colloids, creating an interesting playing field to study phenomena like phase separation, transient gelation, and more exotic cluster formation [2]. Latex colloids that are both sterically and electrostatically stabilized ensure reversibility of the observed phase behaviour, and increase control over the particle stability. We systematically vary salt concentration and polymer size to cover a series of ratios between the ranges of attraction and repulsion. The resulting phase diagrams are compared to the relatively straightforward Generalized Free Volume Theory [3].

[1] Fleer, G. J.; Tuinier, R. Adv. Colloid Interfac. 2008, 143, 1. Bhat, S.; Tuinier, R.; Schurtenberger, P. J Phys-Condens Mat 2006, 18, L339.

[2] Stradner, A.; Sedgwick, H.; Cardinaux, F.; Poon, W. C. K.; Egelhaaf, S. U.; Schurtenberger, P. Nature 2004, 432, 492.

[3] Fortini, A.; Dijkstra, M.; Tuinier, R. J. Phys.-Condens. Mat. 2005, 17, 7783. 
Polymers \& Colloids, Lecture

Tailoring Surface Properties of Fluoropolymers with Structured Polyelectrolyte Brushes

Sonja Neuhaus ${ }^{1}$, Celestino Padeste $^{1}$, Nicholas D. Spencer $^{2}$

${ }^{1}$ Laboratory for Micro and Nanotechnology, Paul Scherrer Institut, 5232 Villigen PSI, Switzerland

${ }^{2}$ Laboratory for Surface Science and Technology, ETH Zurich, 8093 Zurich, Switzerland

We present a strategy to tailor the surface of fluoropolymer foils with welldefined structures of polyelectrolyte brushes. Patterns of radicals serving as polymerization initiators are created on the surface of ETFE foils by exposure to EUV light using the x-ray interference lithography beamline at the Swiss Light Source (SLS). Micro- and nanostructured brushes are grafted from the initiator patterns by free radical polymerization [1]. Polyelectrolyte brushes were obtained in one step for monomers such as methacrylic acid or 4-vinylpyridine, or in two steps as illustrated below for $N$-vinylformamide. The height of the polymer brushes could be tailored by the exposure dose, which determines the initiator density, and conditions of the grafting reaction. The grafting method allows endowing chemically inert and flexible polymer substrates with the wide range of interesting properties of polyelectrolytes, including a $\mathrm{pH}$ dependent charge density along the polymer backbone or specific interactions with metal ions, as illustrated in a selection of examples.

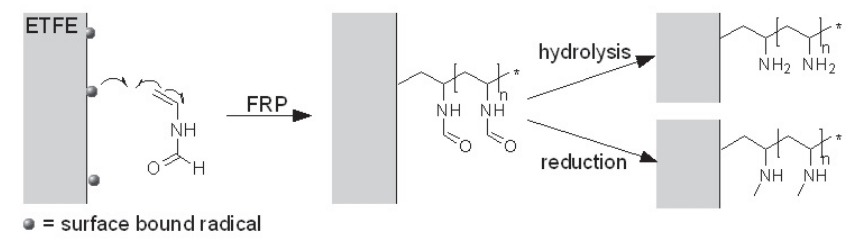

[1] H.-P. Brack, C. Padeste et. al., JACS 2004, 126, 1004.

Polymers \& Colloids, Lecture

\section{Structural control of porous polymeric materials through magnetic Gelation}

Marco Furlan, Bastian Brand, Lattuada Marco and Massimo Morbidelli

ETH Zurich, Institute for Chemical and Bioengineering, Wolfgang Pauli Strasse 10, CH-8093 Zurich, Switzerland

In this work, we introduce a novel procedure to create porous polymeric materials using a process that we named magnetic gelation. We start by preparing magnetic polymer nanoparticles, composed of magnetite nanocrystals dispersed into a polymer matrix, via miniemulsion polymerization. Due to the superparamagnetic behavior of the nanocrystals embedded in the polymer matrix, the nanoparticles prepared in this manner develop strong and reversible dipolar interactions only in the presence of an external magnetic field. Both the size of the nanoparticles and the amount of magnetite nanocrystals encapsulated inside them has been varied, so as to tune the strength of the magnetic interactions. Dispersions containing a few volume percentages of these magnetic nanoparticles, which are stabilized by means of electrostatic interactions, have been partially destabilized through the addition of controlled amounts of electrolytes and their self-assembly and gelation behavior in the presence and in the absence of an external magnetic field have been investigated. In the absence of magnetic fields, the particles self-assemble into random fractal clusters, which eventually percolate to form a colloidal gel. Instead, when an external magnetic field is applied, the particles align themselves in columnar structures in the direction of the field. By tuning the intensity of magnetic interactions, different extents of anisotropy in the final material are obtained. The materials obtained through this magnetic gelation process have been hardened through partial fusion of the nanoparticles and characterized by means of electron microscopy and torque magnetometry.
Polymers \& Colloids, Lecture

Kinetics of fibrillation of beta-lactoglobulin amyloid fibers

Sreenath Bolisetty ${ }^{1}$, Jozef Adamcik ${ }^{1}$, Raffaele Mezzenga ${ }^{{ }^{*}}$

${ }^{1}$ Food and Soft Materials Science, Institute of Food, Nutrition and Health,

ETH Zurich, CH-8092 Zurich, Switzerland.

We investigated the kinetics of formation of multi-stranded betalactoglobulin protein fibrils [1] at $\mathrm{pH} 2$ and $90^{\circ} \mathrm{C}$ by Small angle Neutron Scattering, Static and Dynamic Light Scattering and Atomic Force Microscopy (AFM). Dynamic light scattering clearly demonstrates the conversion of beta-lactoglobulin monomers $(2 \mathrm{wt} \%)$ into semi-flexible protein fibrils on heating at $90^{\circ} \mathrm{C}$. Small Angle Neutron Scattering further supports these results [2]. In addition atomic force Microscopy, allows resolving some details of the fibers at the molecular scale, which bulk scattering techniques cannot capture. Thus, we are able to resolve time-evolving individual stages of the formation of helical pitches along the fibers due to the twisting of different filaments around each other. This ultimately results in multi-strand fibrils formation with periodic cross-sectional fluctuations. Individual stages of fibrillation are discussed in terms of the colloidal physics involved.

[1] Adamcik, J.; Jung, J.M.; Flakowski, J.; Rios, P.D.L, Dietler, G.; Mezzenga, R. Nature Nanotechnology, 2010, ( DOI: 10.1038/NNANO.2010.59).

[2] Jung, J. M.; Savin, G.; Pouzot, M.; Schmitt, C.; Mezzenga, R. Biomacromolecules 2008, 9, 2477.

Polymers \& Colloids, Lecture

Poly(acrylicacid) binding on latex particles

$\underline{\text { Amin Sadeghpour, Andrea Vaccaro, Michal Borkovec }}$

Department of Inorganic, Analytical and Applied Chemistry, University of Geneva, Quai Ernest-Ansermet 30, 1211 Geneva 4, Switzerland.

The complexation of Poly(acrylic acid), PAA, as a weakly charged flexible polyelectrolyte onto an oppositely charged spherical surface is studied. Amidine functionalized latex particles has been titrated by wide range of molecular weight of PAA, studying electrophoretic mobility and suspension stability by different light scattering methods. Effect of different interparticle/particle-polymer interactions such as, surface potential, surface patchiness, polymer bridging and polymer depletion have been considered.

Studying acrylic acid oligomers, incomplete adsorption has been found while the particles surface charge variation behavior, during titration, depends on their concentration. Consequently, the study of binding isotherms of these molecules received the great interest which provides information about adsorbed layer properties and the thermodynamics of complexation phenomena. Electrophoretic mobility measurement with a simple and quick method has been used to estimate the adsorption isotherms considering the effect of $\mathrm{pH}$, ionic strength and chain length. 
Microwave-assisted nonaqueous sol-gel routes to metal oxide nanoparticles

\section{$\underline{\text { Idalia Bilecka, Markus Niederberger }}$}

Department of Materials, Multifunctional Materials, ETH Zürich

Microwave-assisted nonaqueous sol-gel routes became a promising alternative to other liquid-phase methods, offering a one-pot synthesis approach leading to highly crystalline nanoparticles within a few minutes [1]. These solution routes are based on the reaction of metal organic precursors with benzyl alcohol and offer the possibility to tailor the crystal size and shape by choosing the appropriate reaction conditions [2].

It was recently observed that when microwave irradiation was used as a heating media the chemical reaction rate was highly increased, affecting the nucleation rate and the crystal growth [3]. Under such conditions nanoparticle formation is highly dependent on the experimental conditions such as concentration, time and temperature, offering great opportunities for tailoring the crystal size as well as for the development of predictable size-control strategies.

We will present a short overview of the microwave assisted nonaqueous solgel technique comporting the apparatus, the reaction monitoring and the general mechanistic principles of nanoparticle formation. In addition, time dependant crystal growth under microwave irradiation as well as the reaction conditions and their effect on nanoparticle size will be discussed based on $\mathrm{ZnO}$ as example.

[1] I. Bilecka, I. Djerdj and M. Niederberger, Chem. Commun., 2008, 886-888.

[2] M. Niederberger, Acc. Chem. Res., 2007, 40, 793-800.

[3] I. Bilecka, P. Elser, M. Niederberger, ACS Nano. 2009, 3, 2, 467-477.

Polymers \& Colloids

Self-assembly of globular proteins in mixed solvents

$\underline{\text { Ilja K. Voets }}{ }^{1}$, Christian Moitzi ${ }^{1}$, Elizabeth P. G. Areas ${ }^{2}$, and Peter Schurtenberger ${ }^{1}$

${ }^{1}$ Adolphe Merkle Institute, University of Fribourg, Route de l'ancienne Papeterie, CH-1723 Marly 1, Switzerland

${ }^{2}$ Departamento de Química Fundamental, Instituto de Química,

Universidade de São Paulo, Av. Prof. Lineu Prestes 748, CEP 05508-000 São Paulo, SP, Brazil

We are investigating the self-assembly behaviour of the globular protein lysozyme in the solvent mixture dimethylsulfoxide/water as this system has been shown to generate transparent gels above certain critical values of both the DMSO volume fraction, $\varphi_{\mathrm{DMSO}}$ and lysozyme concentration, $[1,2]$ To better understand the origin of this gelation process, we have performed a series of scattering and spectroscopic experiments on dilute, concentrated, and gelled samples.

Three structural regimes have been identified in the ternary system lysozyme/water/DMSO based upon small angle neutron and light scattering experiments: the "folded" $\left(0<\varphi_{\text {DMSO }}<0.7\right)$, "unfolded" $\left(0.7 \leq \varphi_{\text {DMSO }}<\right.$ $0.9)$, and "partially collapsed" $\left(0.9 \leq \varphi_{\mathrm{DMSO}}<1.0\right)$ regime respectively (Figure 1). At intermediate protein concentrations, $C_{\mathrm{LYS}}>10 \mathrm{~g} \mathrm{l}^{-1}$ and high DMSO volume fractions $\left(\varphi_{\mathrm{DMSO}} \geq 0.7\right)$, lysozyme self-assembles into finitesized objects, while gelation is observed at high protein concentrations. Various other globular proteins exhibit similar behaviour in DMSO/water mixtures, but small differences between the various systems were observed. These can be related to structural differences in terms of the amino acid composition and sequence and the amount of disulfide bonds.

[1] M. A. da Silva, E. P. G. Areas, J. Coll. Int. Sci. 2005, 289, 2. [2] E. P. G. Areas, J. A. G. Areas, J. Hamburger, W. L. Peticolas, P. S. Santos, J. Coll. Int. Sci. 1996, 180, 2.

\section{Dielectric elastomers with increased dielectric properties as soft} actuators

\author{
Dorina. M. Opris ${ }^{1}$, Martin Molberg ${ }^{1}$, Christiane Löwe ${ }^{1}$, Frank Nüesch ${ }^{1}$, \\ Patrick Rupper ${ }^{2}$, Daniel Crespy ${ }^{2}$
}

${ }^{1}$ EMPA, Ueberlandstr. 129, CH-8600, Dübendorf, Switzerland

${ }^{2}$ EMPA, Lerchenfeldstr. 5, CH-9014, St. Gallen, Switzerland

Dielectric elastomer actuators (DEA) are made from a thin elastic film sandwiched between two compliant electrodes. When an electric voltage is applied, an electrostatic pressure is acting on the film compressing it in thickness while elongating it in plane (Fig. 1). Electrical energy is thus converted directly into mechanical work. A broad application of DEA is hindered by the required high driving voltage which is in the $\mathrm{kV}$ regime. Several ways can be followed to decrease it, by decreasing the film thickness, decreasing the polymers' elastic modulus, and increasing its dielectric constant ( $\left.\varepsilon^{\prime}\right)$.

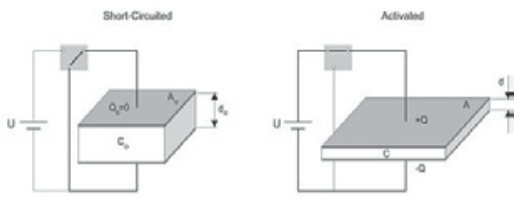

Figure 1. The working principle of EAP: Upon application of a voltage the film in its equilibrium state (left); is compressed (right).

This presentation describes an experimental evaluation on whether or not oligo-phthalocyanines or as-prepared polyaniline (PANI) and poly(divinyl benzene)-encapsulated PANI can be reasonably used as high $\varepsilon$ ' fillers in DEA. ${ }^{1}$

[1] D.M. Opris. F. Nüesch, C. Löwe, M. Molberg, M. Nagel, Chem. Mat 2008, 20, 2668; M. Molberg, D. Crespy, P. Rupper, F. Nüesch, C. Löwe, D. M. Opris, Adv. Funct. Mat. 2010, submitted.

Polymers \& Colloids

Organic electronic materials from oligopeptide-polymer conjugates

$\underline{\text { Roman Marty }}^{1}$, Holger Frauenrath ${ }^{1 *}$

${ }^{1}$ EPFL/Laboratory of Macromolecular and Organic Materials, Institute of Materials, EPFL - STI - IMX - LMOM, Building MXG, Room 040 , Station 12,1015 Lausanne, Email: roman.marty@epfl.ch

Hierarchically structured synthetic polymers containing monodisperse $\pi$ conjugated segments could offer intriguing perspectives for organic electronic devices such as OLEDs, OFETs, and organic solar cells. Our strategy for the preparation of such materials is the synthesis and self-assembly of oligopeptide-polymer conjugates bearing n- and/or p-type semiconducting moieties such as perylene bisimides and oligothiophenes, respectively. Previous investigations in our group revealed that a precise control of position, orientation, and reactivity of $\pi$-conjugated molecular fragments by directional non-covalent interactions, such as $\mathrm{N}-\mathrm{H} \cdots \mathrm{O}=\mathrm{C}$ hydrogen-bonding, is achieved. The investigated macromonomers gave rise to well-defined fibrillar features with a length of several micrometers, and a uniform width of a few nanometers.

[1] Jahnke, E.; Frauenrath, H. Chem. Eur. J. 2008, 14, 2942. 
Polymers \& Colloids

Synthesis and Characterization of Poly(isobutylene)-Oligopeptide Conjugates

Emmanuel Croisier ${ }^{1}$, Su Liang $^{1}$, Holger Frauenrath ${ }^{1 *}$

${ }^{1}$ EPFL/Laboratory of Macromolecular and Organic Materials, Institute of Materials, EPFL - STI - IMX - LMOM, Building MXG, Room 037, Station 12,1015 Lausanne, Email: holger.frauenrath@epfl.ch

Spider dragline silk exhibits outstanding physical properties and has received considerable attention in recent decades. ${ }^{1}$ In order to mimic certain aspect of its molecular and nanoscopic structure, we prepared several poly(isobutylene)-oligopeptide conjugates, which consist of poly(isobutylene) (PIB) as the soft segment and one or two monodisperse, chiral, and $\beta$-sheet-forming terminal oligopeptides as the hard segments, with up to five alanine residues and either Fmoc or acetyl end groups. The properties of these four series of materials, such as their solubility in pure organic solvents and polar-apolar solvent mixtures, their secondary structure formation in the solid state and in organic solvent, as well as their thermal properties, including decomposition, glass transition temperature, and crystallization behavior were investigated and compared. Furthermore, preliminary investigations concerning the film formation and electrospinning were carried out on selected materials. The experimental results show that the formation of antiparallel $\beta$-sheets is determined by both the length of the oligopeptides segments and the end groups. Generally, the acetylated PIBoligopeptide conjugates showed more defined secondary structures both in the solid state and in organic solvents. Correspondingly, they exhibited poorer solubility, higher decomposition temperatures and a stronger preference for crystallization.

[1] F. Vollrath, D. P. Knight, Nature 2001, 410, 541-548

\section{Polymers \& Colloids}

Transparent fabric electrodes for organic photovoltaics W. Kylberg ${ }^{1}$, F. A. Castro ${ }^{1}$, P. Chabrecek ${ }^{2}$, R. Hany ${ }^{1}$, F. Nüesch ${ }^{1}$

${ }^{1}$ Empa, Laboratory for Functional Polymers, Überlandstrasse 129, 8600 Dübendorf, Switzerland

${ }^{2}$ Sefar AG, Printing Solutions, Freibach, 8425 Thal, Switzerland

The development of flexible and transparent electrodes is an important step for the introduction of cheap organic photovoltaics into mass production. We report the use of conducting precision fabrics as transparent and flexible electrode for organic semiconductor-based thin film devices. ${ }^{2}$ Optimized fabrics reach light transmittance over $90 \%$ throughout the visible and near infrared spectra. Surface resistivity is as low as $\sim 3 \mathrm{Ohms} / \mathrm{square}$, which decreases Ohmic losses when scaling up to large area devices. We have applied these electrodes to organic/polymer photovoltaic devices (figure 1) and photoelectric measurements show promising results. It was found that the electrode surface topography was a critical parameter and that the intimate contact at the metal-fibre / polymer interface is essential for improved hole collection.
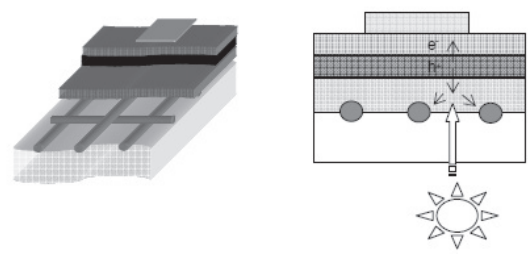

Figure 1 Schematic figure of the organic solar cell design with the fabric fibres as the substrate electrode.

[1] F. C. Krebs, Sol. Energy Mater. Sol. Cells, 2009, 93, 1636-1641

[2] F.A. Castro, P. Chabrecek, F. Nüesch, R. Hany, Phys. Status Solidi $R L L, 2009,3,278-280$
Polymers \& Colloids

Repulsive and attractive electrostatic interactions between sulfate latex particles modified by adsorption of poly(ethylene imine)

\section{Finessi, S. Prashant, P. Maroni and M. Borkovec}

Laboratory of Colloid and Surface Chemistry, Department of Inorganic, Analytical, and Applied Chemistry, University of Geneva, Sciences II,

30 Quai Ernest Ansermet, 1211 Geneva 4, Switzerland

Forces between negatively charged colloidal latex particles in the presence of adsorbed oppositely charged linear poly(ethylene imine) (LPEI) are studied with the colloidal probe technique based on atomic force microscopy (AFM). With increasing adsorbed amount of LPEI, the system passes through an isoelectric point (IEP), and subsequently the surface charge is being reversed. This mechanism is indeed confirmed with the force measurements by the AFM. By varying the adsorbed amount, the strength of these forces can be tuned from repulsive to attractive. At low amounts of adsorbed polymer, the system shows a strong electrostatic repulsion, which decreases until IEP is reached. At the IEP, van der Waals attraction becomes important. Beyond the IEP, the surfaces repel again. At higher adsorbed amount, the surface saturates and the strength of the interactions remains constant.
Polymers \& Colloids

\section{Materials from Oligo(phenylene) Amphiphiles Dragana Paripovic ${ }^{1}$, Holger Frauenrath ${ }^{1 *}$}

${ }^{1}$ EPFL/Laboratory of Macromolecular and Organic Materials, Institute of Materials, EPFL - STI - IMX - LMOM, Building MXG, Room 037, Station 12, 1015 Lausanne, Email: holger.frauenrath@epfl.ch

Materials from conjugated oligomers have attracted interest in the development of organic electronic devices. ${ }^{2}$ The performance of these materials strongly depend on their degree and type of supramolecular organization. Rod-coil systems consisting of flexible coil segments covalently attached to conjugated oligomers as the rigid rod segments provide an excellent means to create well-defined supramolecular self-assemblies. ${ }^{2}$ Ordered phases of oligophenylene dendrimers, on the other hand, have been used as molecular precursors for the preparation of nanostructured carbon materials. ${ }^{3}$

Here, we present the synthesis and characterization of a series of amphiphilic rod-coil molecules based on oligo(phenylene)s as the hydrophobic rod segments and oligo(ethylene oxide)s as flexible, hydrophilic coil segments. We investigated their molecular spectroscopic and their thermal properties in bulk and in aqueous solution, their propensity to form lyotropic liquid-crystalline phases, and their potential to be used as precursors in the preparation of novel types of mesoscopically structured carbon-rich materials.

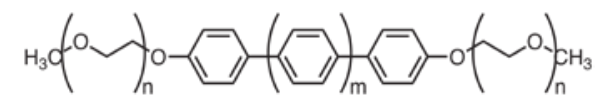

[1] Qi-feng Zhou, Chin.J. Polym. Sci, 2006, 24, 63-98

[2] M. Lee, Ja-Hyoung Ryn, Struct. Bond (Berlin), 2007, 128, 63-98

[3] K. Müllen, Adv. Mater, 2005, 17, 1492-1496 
Polymer Brush Guided Preparation of Thin Gold Films

\section{Dusko Paripovic $^{1}$, Harm-Anton Klok ${ }^{1}$}

${ }^{1}$ École Polytechnique Fédérale de Lausanne (EPFL), Institut des Matériaux, Laboratoire des Polymères, Bâtiment MXD, Station 12, CH-1015 Lausanne

Deposition of thin noble metal films is performed in microelectronic industry by several methods including chemical and physical vapor deposition and electroplating. Although routinely used, these methods suffer from several drawbacks such as need for complicated and costly setups, difficulty in finding volatile precursors, restrictions with the respect to substrate's geometry and composition etc. Polymer brushes, with their intriguing properties, emerged in recent years as a new promising tool for deposition of thin inorganic films [1][2].

Here we exploit polymer brushes as matrixes for preparation of nanoscopically thin gold films. The preparation process (scheme 1) comprises (i) synthesis of positively charged polymer brushes, (ii) loading the polymer brush matrix with the gold precursor, (iii) its reduction to elemental gold and finally (iv) removal of polymer brushes by oxygen plasma. We have demonstrated that the thickness of such gold films can be controlled by the thickness of the initial polymer brush matrix. In addition, the influence of process parameters on film's final morphology will be presented.

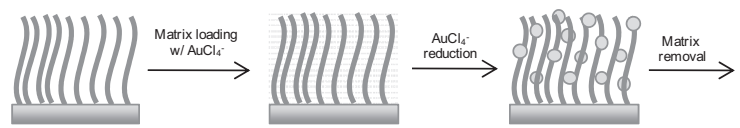

Scheme 1. Preparation of thin gold film via polymer brushes matrix

[1] S. Tugulu, H-A. Klok, Angew. Chem. Int. Ed. 2006, 45, 7458.

[2] D. J. Kim et al., Langmuir 2004, 20, 7904.

Polymers \& Colloids

Polymer materials containing oligothiophene segments

Damien Rolland $^{1}$, Holger Frauenrath ${ }^{1}$

${ }^{1}$ EPFL / Laboratory of Macromolecular and Organic Materials, EPFL-STIIMX-LMOM, Station 12, 1015 Lausanne

Oligothiophene is of one of the most interesting p-type organic semiconductor ${ }^{[1]}$ The control of the macromolecular organization of organicsemiconductive materials is a key for tuning their optoelectronical properties. ${ }^{[2]}$ The use of advanced polymer synthesis and of polymer processing provides useful tools to develop new materials containing nanostructured oligothiophenes.

In this work, we investigated the synthesis and characterization of novel oligothiophene $\alpha, \omega$-functionalized with bulky activated esters. These terminal groups, at the same time, provide very good solubility in common organic solvents and allow polymer formation through amide or ester bonds Its excellent solubility facilitates its handling for polycondensation. Thus, the synthesis of poly(amide) thermoplasts and corresponding hard-soft block copolymers starting from these novel oligothiophene derivatives has been investigated.

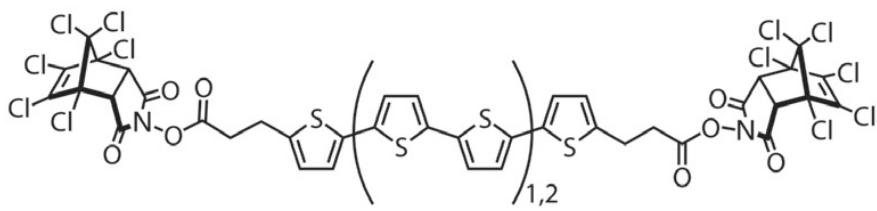

[1] Amanda R. Murphy, Jean M. J. Fréchet, Chem. Rev. 2007, 107, 1066.

[2] Francis Garnier, Acc. Chem. Res. 1999, 32, 209.
Influence of the Backbone Structure on the Release of Bioactive Volatiles from Maleic acid Based Polymer Conjugates

Damien L. Berthier, Alain Trachsel, and Andreas Herrmann

${ }^{1}$ Firmenich SA, Materials Science Department, R\&D, Route des Jeunes, 1, 1211 Genève

The controlled release of bioactive molecules has become a major area of interdisciplinary research in life-sciences. In particular, precursor-type structures have been proven to efficiently deliver bioactive compounds in various applications $[1,2]$. Amphiphilic poly(maleic acid monoester)-based $\beta$ mercapto ketones were synthesized and investigated as potential delivery systems for the controlled release of bioactive, volatile $\alpha, \beta$-unsaturated enone $\delta$-damascone (1) by retro 1,4-addition (Scheme) [3].

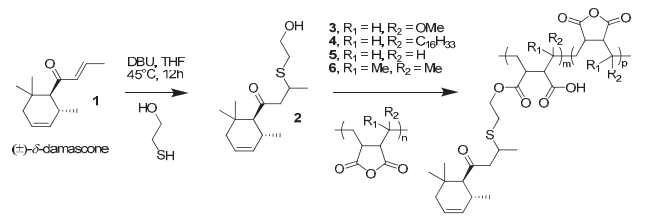

Scheme: One-pot synthesis of poly(maleic acid)-based $\delta$-damascone

In the presence of a cationic surfactant, copolymers were transferred from an aqueous medium to a cotton surface. The deposition of copolymers was measured by fluorescence analysis and the release of $\mathbf{1}$ as a function of time was investigated by dynamic headspace analysis. A good balance of the hydrophobicity and hydrophilicity of the polymer backbone is the key factor to maximize deposition and to optimize release of the bioactive volatiles.

[1] Berthier, D.; Trachsel, A.; Fehr, C.; Ouali, L.; Herrmann, A. Helv. Chim. Acta 2005, 88, 3089.

[2] Berthier, D.; Herrmann, A.; Ouali, L.; Reichlin, D. Firmenich\&Cie Patent WO2007007216

[3] Berthier, D.; Herrmann, A. Firmenich\&Cie Patent WO2008044178

Polymers \& Colloids

Ellipsoidal Silica-Coated Hematite Nanoparticles for Inorganic-Organic Elastomer Nanocomposites

$\underline{\text { Antoni Sánchez-Ferrer }^{1}}{ }^{,}$Mathias Reufer ${ }^{2}$, Raffaele Mezzenga $^{1}$ Peter Schurtenberger ${ }^{2}$, Hervé Dietsch ${ }^{2}$

${ }^{1}$ ETH Zurich, Institute of Food, Nutrition \& Health Schmelzbergstrasse 9, 8092 Zurich, Switzerland

${ }^{2}$ Adolphe Merkle Institute and Fribourg Center for Nanomaterials Route de l'Ancienne Papeterie, PO Box 209, 1723 Marly 1, Switzerland

Magnetic inorganic-organic network nanocomposites are covalently crosslinked polymeric systems, which can be found either in swollen state (magnetic gels) or in dry state (magnetic thermoplastics, thermosets or elastomers), and exhibit magnetic nanoparticles dispersed into the polymer matrix [1]. These materials have the combined advantages of organic polymers (flexibility and processability) and the inorganic nanoparticles (magnetic, optical and electrical properties) [2]. We describe here an in situ procedure for the integration of surface modified hematite nanoparticles that can act as covalent crosslinkers in the final elastomeric network [3]. For the first time the use of silica-coated hematite $(\mathrm{SCH})$ nanoparticles as crosslinkers has been reported and the analysis of their hybrid nanocomposite (E1) properties presented. We compare this system with systems with non-crosslinkable nanoparticles (E2), and with pure elastomeric networks (E0) made of the same polymer backbones (Figure 1).

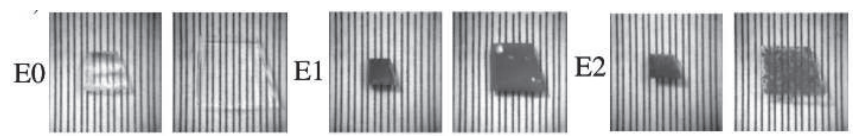

Figure 1. Pictures of the unswollen and swollen dimensions for the three networks

[1] M. Zrínyi, Trends Polym. Sci. 1997, 5, 280.

[2] H. Althues, J. Henle, S. Kaskel, Chem. Soc. Rev. 2007, 36, 1454.

[3] A. Sánchez-Ferrer, M. Reufer, R. Mezzenga, P. Schurtenberger, H. Dietsch, Nanotechnology 2010, 21, in press. 
Polymers \& Colloids

Study of the formation of photochemically active organic multilayer films by surface plasmon resonance, ellipsometry and quartz crystal microbalance.

Mariya Porus, Plinio Maroni, Naomi Sakai and Michal Borkovec

University of Geneva, Science II, 30 Quai Ernest-Ansermet, 1211 Geneva, Switzerland

The present study is focused on the characterization of formation of photochemically active organic films on gold surface. These films are formed by sequential adsorption of positively and negatively charged naphtalenediimide derivatives (NDIs) of p-oligophenyls (POP) and oligophenylethynyls (OPE) on gold. Organic films formed with these macromolecules can be considered as sophisticated $\mathrm{n} / \mathrm{p}$-heterojunction systems, where polyaromatic rods play the role of $h^{+}$-transporting elements, while naphtalenediimides of $e^{-}$transporting $\pi$-stacks. Regarding the initiator used to activate gold surface two types of architectures of organic films are expected - "zipper " assembly with POP or OPE rods orienting perpendicular to the surface and "layer-by-layer" assembly, with parallel orientation of rods. Previously it was reported that these constructions show different photochemical activity [1].

Surface plasmon resonance, scanning angle ellipsometry and quartz crystall microbalance were performed to study the formation of POP-NDI and OPENDI films. The monotonous growth of the film in both cases was observed. Refractive indexes of the films as well as their thicknesses in wet and dry state were evaluated using SPR on water/gold and air/gold interfaces. Thickness increment per theoretical layer was estimated to be $1.5 \mathrm{~nm}$ and $1.9 \mathrm{~nm}$ (in dry state) and $3.4 \mathrm{~nm}$ and $3.6 \mathrm{~nm}$ (in wet state) for POP- NDI and OPE-NDI film respectively. By combining SPR and QCM-D water content in organic film was calculated. No difference between two molecular assemblies was detected so far.

[1] Bhosale R., Perez-Velasco A et al., Angew. Chem. Int. Ed. 2009, 48, 6461-6464.

Polymers \& Colloids

\section{Adsorption of linear polyethyleneimine on charged latex particles}

István Szilágyi, Josè Hierrezuelo, Andrea Vaccaro, Michal Borkovec

Department of Inorganic, Analytical and Applied Chemistry, University of Geneva, 30 Quai Ernest-Ansermet, 1211 Geneva

Polyelectrolytes have been used in several industrial and biological processes; however, their adsorption on colloidal particles has a significant importance in these applications [1]. In the present work, surface charge properties and colloid stability in sulfate functionalized polystyrene latex particle (SL, $\mathrm{R}_{\mathrm{h}}=135 \mathrm{~nm}$ ) dispersions have been studied in the presence of linear polyethyleneimines (LPEI, MW $=2.5-250 \mathrm{kDa}$ ) by electrophoretic mobility and dynamic light scattering (DLS) measurements.

At sufficiently low pH (4.0) LPEIs behave as positively charged polyelectrolyte due to the protonation of amino groups. Accordingly, electrophoretic mobility measurements showed that the negatively charged sulfate latex particles can be neutralized (isoelectric point (IEP)) and also overcharged by the adsorption of LPEI on their surface. The effect of these charging phenomena depends on the ionic strength (adjusted by $\mathrm{KCl}$ ) and also the molecular weight of LPEIs. Accordingly, IEPs are shifted toward higher polymer doses with increase of $\mathrm{KCl}$ concentration and decrease of molecular mass of LPEIs.

These results have been confirmed with time-resolved DLS experiments by measuring the aggregation rate of particles at different SL to LPEI ratio and $\mathrm{KCl}$ concentrations. In addition, slow aggregation was observed at sufficiently low and high polymer doses (before and after IEP), whereas fast aggregation occurred near the IEP indicating the absence of repulsive electrical double layer due to charge neutralization and the dominance of the attractive van der Waals forces. Finally, hydrodynamic layer thickness of the adsorbed polymer coat has been determined by DLS and found to be $(6 \pm 0.3)$ $\mathrm{nm}$ at sufficiently high $\mathrm{KCl}$ concentrations (>0.1 M).

[1] M. Borkovec, G. Papastavrou, Curr. Opin. Colloid Interface Sci. 2008, 13,429 .
Polymers \& Colloids

Understanding amyloid aggregation by statistical analysis of atomic force microscopy images

Jozef Adamcik $^{1}$, Jin-Mi Jung ${ }^{2}$, Jérôme Flakowski ${ }^{3}$, Paolo De Los Rios ${ }^{3}$, Giovanni Dietler ${ }^{4} \&$ Raffaele Mezzenga ${ }^{1}$

${ }^{1}$ ETH Zurich, Food \& Soft Materials Science, Institute of Food Nutrition \& Health, LFO23, Schmelzbergstrasse 9, 8092 Zürich, Switzerland ${ }^{2}$ Department of Physics and Fribourg Center for Nanomaterials, University of Fribourg, Ch. Musée 3, CH-1700, Fribourg, Switzerland

${ }^{3}$ Laboratoire de Biophysique Statistique, Ecole Polytechnique Fédérale de Lausanne (EPFL), CH-1015 Lausanne, Switzerland

${ }^{4}$ Laboratoire de Physique de la Matière Vivante, Ecole Polytechnique Fédérale de Lausanne (EPFL), CH-1015 Lausanne, Switzerland

Aggregation of proteins is central to many aspects of daily life, ranging from blood coagulation, to eye cataract formation disease, food processing, or prion-related neurodegenerative infections. In particular, the physical mechanisms responsible for amyloidosis, the irreversible fibril formation of various proteins implicated in protein misfolding disorders such as Alzheimer, Creutzfeldt-Jakob or Huntington's diseases, have not yet been fully elucidated. In this work we demonstrate that by performing a statistical polymer physics analysis on the molecular conformations of amyloid fibrils revealed by single molecule AFM, different stages of amyloidal aggregation can be individually resolved [1]. We present results for aggregates of heatdenaturated $\beta$-lactoglobulin and univocally demonstrate their analogies with amyloid fibrils. These $\beta$-lactoglobulin fibrils have a multi-strand helical shape with twisted ribbon-like structures and we present theoretical and numerical arguments supporting these findings. Our results also suggest a possible general model for amyloid fibril assembly and illustrate the potential of this approach for investigating fibrillar system.

[1] J. Adamcik, J.M. Jung, J. Flakowski, P. De Los Rios, G. Dietler, R. Mezzenga, Nat. Nanotechnol. 2010, doi:10.1038/nnano.2010.59

Polymers \& Colloids

553 Synthesis and "migration" of silver nanoparticles in polyelectrolyte
matrix

Jérôme Girard, Katharina M. Fromm

University of Fribourg, Chemistry department, Switzerland

Nowadays, a wide variety of composite materials consisting of polymers containing metal nanoparticles have been extensively investigated to realize their potential applications ranging from optoelectronics to biomaterials. Nanostructured materials consisting of silver nanoparticles (Ag-NPs) embedded in polymeric matrices show physico-chemical, optical and antibacterial properties $[1,2]$. A simple method to prepare a $\mathrm{AgNP} /$ polyelectrolyte composite was successfully used. Thus, Ag-NPs were obtained through spontaneous formation of nanostructured silver from an $\mathrm{Ag}_{2} \mathrm{O} /$ polystyrene sulfonate (PSS) solution. The nanoparticle size was determined by SEM., and related morphology was investigated by X-ray diffraction techniques. Nanocrack formation was observed during the ageing of the polymer, these cracks were spontaneously filled by silver. The synthesis of the Ag-NP/PSS composite was performed by mixing a PSS solution in water with a $\mathrm{Ag}_{2} \mathrm{O}$ solution in diluted ammonia during 48 hours.

[1] Kirsten Belser, Tünde Vig Slenters, Conelious Pfumbidzai, Grégory Upert, Laurent Mirolo, Katharina M. Fromm, Helma Wennemers, Angew. Chem. Int. Ed. Eng. 2009, 48(20), 3661

[2] Alexandre Mantion, A. Geraldine Guex, Annette Foelske, Laurent Mirolo, Katharina M. Fromm, Monika Painsi, Andreas Taubert, Soft Matter. 2008, 4(3), 606 
Polymers \& Colloids

\section{Styrene / methacrylonitrile co-grafted membranes for fuel cells}

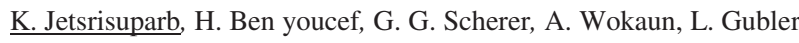

Electrochemistry Laboratory, Paul Scherrer Institut, CH-5232 Villigen PSI, Switzerland

The aging of polymer materials used as proton exchange membrane (PEM) leads to premature failure of the membrane electrode assembly due to chemical and mechanical degradation in the fuel cell environment [1]. Our research is devoted to enhance the chemical and mechanical stability of PEMs for fuel cell application prepared by radiation induced graft copolymerization. Styrene and methacrylonitrile (MAN) are co-grafted into an inert poly(ethylene-alt-tetrafluoroethylene) (ETFE) base film of $25 \mu \mathrm{m}$ thickness and subsequently sulfonated. Improvement in $\mathrm{H}_{2} / \mathrm{O}_{2}$ fuel cell performance and durability was achieved with ETFE- $g$-styrene/MAN membrane compared to ETFE- $g$-styrene membrane at $80^{\circ} \mathrm{C}$ [2].

We have investigated the kinetics of styrene / MAN co-grafting into preirradiated ETFE base film and the influence of the content of styrene and MAN in the grafted membrane on the ex situ fuel cell relevant properties. As the styrene unit carries the sulfonic acid group, the ion exchange capacity, water uptake, and proton conductivity increase with the styrene / MAN ratio in the graft component at fixed graft level. A careful selection of the monomer content is critical for optimizing the conductivity and the dimensional stability of ETFE- $g$-styrene/MAN membranes.

[1] H. Tang, S. Peikang, S.P. Jiang, F. Wang, M. Pan, J. Power Sources 2007, 170, 85-92.

[2] H. Ben youcef, L. Gubler, S. Alkan-Gürsel, D. Henkernsmeier, A. Wokaun, G.G. Scherer, Electrochem. Commun. 2009, 11, 941-944.

Polymers \& Colloids

\section{Nanostructured Carbon Films from Molecular Precursors}

\section{S. Schrettl ${ }^{1}$, T. N. Hoheisel ${ }^{2}$, H. Frauenrath ${ }^{1 *}$}

${ }^{1}$ École Polytechnique Fédérale de Lausanne (EPFL) Laboratory of Macromolecular and Organic Materials Building MXG, Station 12, 1015 Lausanne, Switzerland ${ }^{2}$ ETH Zürich

Department of Materials

Nanostructured carbonaceous materials offer intriguing perspectives for emerging technologies such as hydrogen or lithium storage ${ }^{[1]}$ Our strategy for the preparation of such materials is based on the synthesis, selfassembly, and subsequent carbonization of amphiphilic oligo(ethynylene)s (Figure 1).
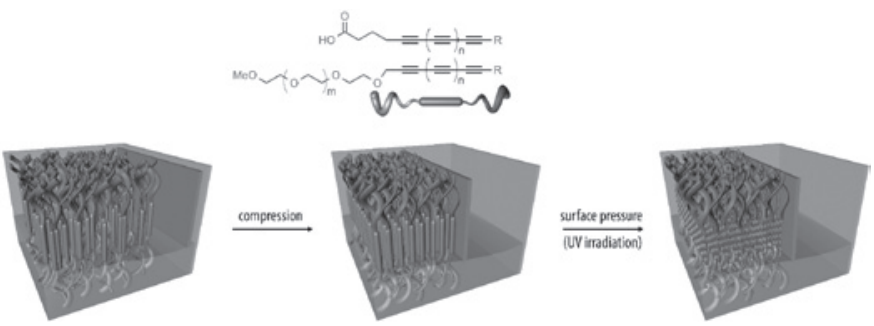

Figure 1. Conceptual approach toward nanostructured carbon materials.

We prepared acid-based and oligo(ethylene oxide)-based oligo(ethynylene) amphiphiles which resemble typical fatty acids and Brij amphiphiles, respectively. The aggregation of these amphiphilic molecules at the air-water interface as well as their reactivity toward carbonization upon UV irradiation in the aggregated state are subject of our investigations.

[1] T. N. Hoheisel, S. Schrettl, R. Szilluweit, H. Frauenrath, Angew. Chem. Int. Ed., 2010, accepted.
Polymers \& Colloids

\section{Sintering of Core-shell Ag/Glass Nanoparticles}

Aline C.C. Rotzetter, Norman A. Luechinger, Evagelos K. Athanassiou, Dirk Mohn, Fabian M. Koehler, Robert N. Grass and Wendelin J. Stark*

* Department of Chemistry and Applied Biosciences, Institute for Chemical and Bioengineering, ETH Zurich, CH-8093 Zurich, Switzerland

Highly conducting metal/glass/ceramic composites were synthesized by pressing and sintering silver-core/ silica glass-shell nanoparticles produced by flame spray synthesis. Compared to other, non-flame synthesis methods leading to core-shell structures, the presented method is more rapid and the glassy matrix can be easily modified. This allowed the preparation of silver core structures with two distinct glassy shells strongly differing in their glass transition temperature. The resulting metal/glass/ceramic composites had a fully homogenous appearance and exhibited an electrical conductivity increase of $\sim 7$ orders of magnitude. Scanning electron micrographs showed clearly the formation of a highly conductive percolated silver network embedded in a ceramic matrix. The Figure shows a scanning electron micrograph of the sintered highly conducting composite treated with hydrofluoric acid (c) and nitric acid (b).

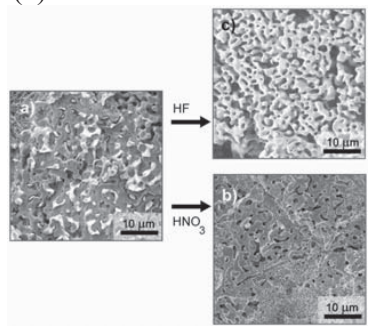

Sintering of core-shell Ag/glass nanoparticles: Metal percolation at the glass transition temperature yields metal/glass/ceramic composites, Aline C.C. Rotzetter, Norman A. Luechinger, Evagelos K. Athanassiou, Dirk Mohn, Fabian M. Koehler, Robert N. Grass (under Review).

Polymers \& Colloids

Stability limits of liquid bridges between colloidal particles.

\section{$\underline{\text { David Megias-Alguacil }}^{1}$, Ludwig J. Gauckler ${ }^{1}$}

${ }^{1}$ ETH-Zürich, Non-Metallic Inorganic Materials, 8093 Zürich

A liquid bridge is essentially a small amount of liquid binding two solid particles, wetting them partially. The bridges may be concave or convex, whose meniscus contour may be approximated by an arc-of-circumference. The limiting surface-to-surface distances, $H_{\min }$ and $H_{\max }$, between particles of radius $R$ are determined by $V_{\text {rel }}=V_{\text {liquid }} / V_{\text {sphere }}$, and the contact angle, $\theta$.

For a concave meniscus, $\theta<90^{\circ}$, and $H_{\min }$ [1] and $H_{\max }$ [2] are given by:

$H_{\text {min }}=2 R\left[\frac{2 V_{r e l}+(1-\sin \theta)^{2}(2+\sin \theta)}{3 \cos ^{2} \theta}-(1-\sin \theta)\right]$

$H_{\max }=R(1+\theta / 2) \sqrt[3]{4 / 3 \pi V_{r e l}}$

For the case of convex bridges, when $\theta<90^{\circ}, H_{\min }$ is [3]:

$V_{r e l}=\frac{3}{2}\left\{\left(1-\left(\frac{H_{\min }}{2 R}+1\right) \cot \theta\right)^{2}\left(\frac{H_{\min }}{2 R}+1\right)+\left(\frac{H_{\min }}{2 R}+1\right)^{3}\left(\cot ^{2} \theta+\frac{2}{3}\right)-\right.$

$\left.-2 / 3+\left(1-\left(H_{\min } / 2 R+1\right) \cot \theta\right)\left(H_{\min } / 2 R+1\right)^{2}\left[\cot \theta+\theta\left(1+\cot ^{2} \theta\right)\right]\right\}(3)$

$H_{\max }$ is equal to Eq.(1) because the convex-concave transition. When $\theta>90^{\circ}$, $H_{\min }$ and $H_{\max }$ are given, respectively, by the conditions [3]:

$H_{\min }=2 R(\rho / R-1)$

being $\rho$ the radius of curvature of the meniscus, and:

$V_{r e l}=3 / 2\left(H_{\max } / 2 R\right)^{3}\left[2 / 3+3 \tan ^{2} \theta+(\theta-\pi / 2) \tan \theta\left(1+\tan ^{2} \theta\right)\right]$

[1] D. Megias-Alguacil, L.J. Gauckler, AIChE J. 2009, 55,1103.

[2] G.Lian, C.Thornton, M.J.Adams, J.Colloid Interf. Sci. 1993, 161, 138.

[3] D. Megias-Alguacil, L.J. Gauckler, Powder Tech. 2010, 198, 211. 
Polymers \& Colloids

\section{Covalent and efficient attachment of ligands to polymer vesicles}

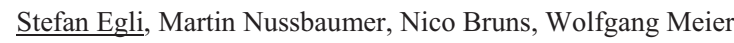

Department of Chemistry, University of Basel, Klingelbergstrasse 80, 4056 Basel, Switzerland

During the last decade the interest in polymer vesicles for applications in fields like drug delivery and sensor technology increased rapidly ${ }^{1}$. We improved the conjugation chemistry at the vesicle membrane surface towards covalent and controllable attachment of targeting moieties like peptides, oligonucleotides or proteins to polymer vesicles.

Using vesicles prepared of different ratio of two poly(dimethylsiloxane)-bpoly(2-methyloxazoline) polymers, one having hydroxyl and the other secondary amine terminal functionalities, we studied the covalent attachment of a succinimidyl ester activated fluorescent dye. By fluorescence correlation spectroscopy (FCS) we could see a clear trend towards lower fluorescence intensity per particle by having lower ratio of amine functionalized polymer in vesicles. Furthermore we functionalized vesicles with succinimidyl 4formylbenzoate (S-4FB) and enhanced yellow fluorescent protein (eYFP) with succinimidyl 6-hydrazinonicotinamide acetone hydrazone (S-HyNic) ${ }^{2}$. Vesicles and eYFP were incubated in buffer $\mathrm{pH} 6$ at room temperature over night resulting in eYFP-vesicle conjugates connected by a stable bis-aryl hydrazone bond. We performed diffusion measurements by FCS from which we calculated an average hydrodynamic radius for the eYFP-vesicle conjugates of $106 \mathrm{~nm}$.

Since this conjugation system is very specific, efficient and works without any toxic conjugation agents, we introduced a new and simple method to covalently attach biomolecules to polymer vesicles.

[1] Onaca et al., Macromol. Biosci. 2009, 9, 129.

[2] Bruns et al., Angew. Chem., Int. Ed.. 2009, 48, 5666.

Polymers \& Colloids

Protein Coupled Copper-Catalysts for Atom Transfer Radical Polymerization in Pure Water

$\underline{\text { Kasper Renggli, Farzad Seidi, Nico Bruns }}$

Department of Chemistry, University of Basel, Klingelbergstrasse 80, 4056 Basel, Switzerland

Atom transfer radical polymerization (ATRP) has emerged as one of the most powerful synthetic techniques in polymer science. Similarly to other controlled radical polymerization (CPR) methods, it allows the synthesis of polymers with predetermined molecular weight, narrow molecular weight distribution, as well as desired composition and molecular architecture. ATRP is a metal complex-mediated reaction, with copper-based catalysts being most commonly used. Recent advances have provided access to polymers with protein-reactive end groups synthesized by ATRP and other CPR methods. Conjugates have also been prepared by polymerizing directly from the proteins. To the best of our knowledge, preparation of protein catalyst conjugates for ATRP have not yet been reported.

Here we present an easy and efficient way to couple $\mathrm{Cu}$-based catalysts to BSA, a serum albumin protein, and other proteins. The resulting nanoscale catalyst enables ATRP in pure water and shows improved performance compared to conventional, low molecular weight catalysts.

[1] N. V. Tsarevsky and K. Matyjaszewski, Chem. Rev. 2007, 107, 22702299.
Polymers \& Colloids

Protein-Mediated Indication of Damage in Polymers and FiberReinforced Composites

Nico Bruns, Samuel Lörcher, Urs Siegenthaler

Department of Chemistry, University of Basel, Klingelbergstrasse 80, 4056 Basel, Switzerland

Living organisms have developed ways to detect and report damage of their tissue. This is usually accomplished by nerve signals, e.g., in the form of pain, or by optical signals, e.g., by the dark red color of a bleeding wound. The indication of damaged tissue is crucial for an organism in order to start to protect the respective body part. In technical applications, polymer-based materials are often used as load-bearing materials because they are lightweight, possess excellent mechanical properties and are easy to manufacture into any desired shape. Fiber-reinforced composites in particular find application in the aerospace, the automotive and the sporting goods sector. However, common polymers and composites do not visualize damage to alert the user of impeding danger. In order to avoid accidents, man-made polymeric materials that detect and report small scale structural damage before catastrophic failure occurs are highly desirable.

We have doped polymers and fiber-reinforced composites with engineered proteins. Microcracks, delamination defects and other damages are reported by the proteins in the materials through a local change in the fluorescent properties of the proteins, e.g. a change in fluorescence resonance energy transfer (FRET) [1] or the vanishing of fluorescence. These signals can be observed by fluorescence microscopy and other fluorescence methods.

[1] N. Bruns, K. Pustelny, L. M. Bergeron, T. A. Whitehead, D. S. Clark, Angew. Chem. 2009, 121, 5776; Angew. Chem. Int. Ed. 2009, 48, 5666.

Polymers \& Colloids

CW and Pulse EPR Spectroscopy on Polymer Clay Nanocomposites

Udo Kielmann, Inés Garcia Rubio, Gunnar Jeschke

ETH/Laboratory of Physical Chemistry, Wolfgang-Pauli-Strasse 10, 8093 Zurich, Switzerland

Polymer clay nanocomposites consist of a polymer film with inclusions of silicate nanoparticles. The interest from scientists and industry comes because of their superior properties compared to those of the pure polymers. Studies on detailed structure at the nanoscale range are still rare, although they would help to understand the interactions responsible for the improvement of their properties. EPR spectroscopy together with spin probe techniques can be useful to understand these complex systems by using the sensitivity of the spectra to the dynamics and local environment of the probe.

Here we present an EPR study of different nanocomposites, produced by mixing an aqueous micellar dispersion of polymer with exfoliated clay and subsequent drying or precipitation with salts and annealing at $50{ }^{\circ} \mathrm{C}$ to obtain composite films. We used SDS-stabilized NBR and PMMA-co-BA dispersions as well as different disk-shaped clay nanoparticles. Spin probes with different functionalities as TEMPO-derivatives (CAT-1) and DOXYLstearates were added aiming different locations in the nanocomposites.

CW EPR spectroscopy was applied to dispersions and films to get insights into their structural and dynamic properties. We show that Cat-1 is preferentially found on the clay surface, while DOXYL-stearates associate with the polymer particles. The CW EPR spectra of the probes are sensitive to the interaction between clay particles and polymer particles in dispersion. Their rotational correlation time in the dispersions varies between different silicate types or polymers. This can also be observed for the DOXYL-stearate films in a wide temperature range. We used pulse EPR methods (ESEEM, ENDOR, HYSCORE) to study the hyperfine structure for investigation of the local structure of the clay polymer nanocomposites. 


\section{Synthesis of Novel Polymers for the Production of Polymeric Optical} Fibers

\author{
Evren Aslan Gürel ${ }^{1}$, Laura Gottardo ${ }^{(2)}$, Lukas J. Scherer ${ }^{(3)}$, Rudolf \\ Hufenus $^{(4)}$, René M. Rossi ${ }^{(5)}$
}

${ }^{(1-5)}$ EMPA, Swiss Federal Laboratories for Materials and Research, Lerchenfeldstr. 5, 9014, St.Gallen, Switzerland

Polymeric optical fibers (POF) have found a wide range of applications in medicine, civil engineering and construction ${ }^{1}$. Polymeric optical fibre fabrics behave as "a second skin" and detect vital signals of the human's body or variations in the wearer's environment. The focus of this contribution is the synthesis and characterization of polymethylmethacrylate copolymers ${ }^{2}$. In order to gain flexibility in the end-product, methyl methacrylate is copolymerized with different acrylic monomers (Figure 1).

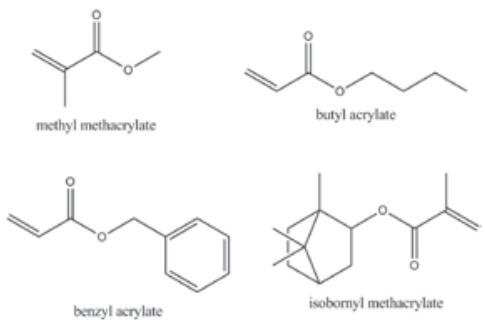

Fig 1: Molecular structures of used monomers

Suspension polymerization is chosen due to easily up-scale. Chemical structures, morphologies, mechanical and thermal properties are determined and different copolymer structures are compared. Melt spinnability of the novel polymers is studied and light attenuation of as-produced fibers are measured.

[1] El-Sherif, M. A. et al., A. J. Intel. Mat. Styst. Str., 2000, 11(5), 407

[2] Park, S. et al., Macromol Res. 2007, 15(5), 418

Polymers \& Colloids

\section{The largest synthesized structure-defined linear macromolecule}

\section{Baozhong Zhang, A. Dieter Schlüter}

ETH Zürich, Wolfgang-Pauli-Strasse 10, 8093 Zürich, Switzerland

Thickness of linear chains is ill-defined and rarely studied in polymer chemistry and physics. For a comprehensive study of this aspect, it has been recently developed in our group a synthetic attach-to protocol that allows the cross-section diameter of polymer chains to be systematically tuned by a consecutive dendronization. ${ }^{[1]}$ The structure perfection of the resulting polymers, which are so-called dendronized polymers (denpols), was determined by quantitative UV measurements. ${ }^{[2]}$ The AFM images of denpols with different generations indicate differences in chain stiffness and apparent heights. Recently a few unprecedented observations could be correlated to the unique thickness effect. It has been reported that dendronized polymers can back fold to form duplexes upon peripheral charging. ${ }^{[3]}$ In a recent AFM pulling experiment, it has been observed that the persistence lengths and stretching elasticity of charged denpols depend on the generation. ${ }^{[4]}$ These phenomena are not known for conventional "thin" polymers.

By this highly efficient and precise synthetic methodology, we are currently able to produce the largest synthetic linear macromolecule that has a comparable molar mass, cross section size and shape as plant viruses.

[1] Y. Guo, J. D. van Beek, B. Zhang, M. Colussi, P. Walde, A. Zhang, A. Halperin, M. Kröger, A. D. Schlüter, J. Am. Chem. Soc. 2009, 131, 11841 . [2] L.Shu, I. Gössl, J. P. Rabe, A. D. Schlüter, Macromol. Chem. Phys. 2002, 203, 2540 .

[3] W. Zhuang, E. Kasëmi, Y. Ding, M. Kröger, A. D. Schlüter, J. P. Rabe,. Adv. Mater. 2008, 20, 3204

[4] I. Popa, B. Zhang, P. Maroni, A. D. Schlüter, M. Borkovec, Angew. Chem. Int. Ed. 2010, accepted.
Enzyme immobilization on a $\mathrm{SiO}_{2}$ surface with the help of a dendronized polymer via avidin biotin linkage

\author{
Sara Fornera $^{1}$, Tobias Balmer ${ }^{2}$, Peter Walde $^{1}$
}

${ }^{1}$ Polymer Chemistry, Department of Materials, ETH Zürich, WolfgangPauli-Str. 10, CH-8093 Zürich, Switzerland

${ }^{2}$ Laboratory for Surface Science and Technology, Department of Materials,

ETH Zürich, Wolfgang-Pauli-Str. 10, CH-8093 Zürich, Switzerland

Efficient enzyme immobilization on a surface is often the crucial step in the preparation of a biosensor. We have immobilized a model enzyme, horseradish peroxidase (HRP), via a biotin avidin dendronized polymer system on a $\mathrm{SiO}_{2}$ surface. Dendronized polymers (denpols) are polymers where every repeating unit carries a dendrimer with, ideally, a well defined chemical structure [1]. The denpol selected was water soluble and decorated with amino groups. Some of these amino groups were selectively modified with biotin. Then, in the first adsorption phase, the biotinylated polymer was adsorbed on a $\mathrm{SiO}_{2}$ surface. The specificity of the avidin-biotin interaction on the surface was proven and biotinylated HRP was immobilized. The adsorption processes were monitored by the transmission interferometric adsorption sensor (TInAS) [2]. Preliminary results showed that the immobilized enzyme kept its ability of oxidizing substrate molecules in the presence of hydrogen peroxide.

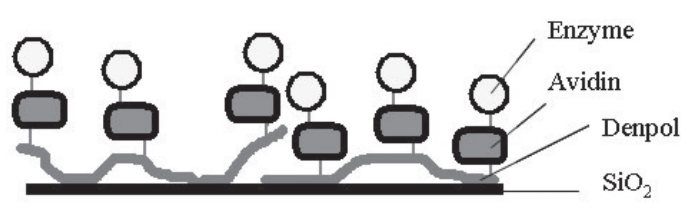

[1] A. D. Schlüter, Top. Curr. Chem. 2005, 245, 151.

[2] M. Heuberger, T. E. Balmer, J. Phys. D: Appl. Phys. 2007, 40, 7245.

Polymers \& Colloids

Can reversible adhesion be achieved between oppositely charged surfaces?

\section{Thomas Wermelinger, Achim Ecker, Sandra Uebersax, Raffaela Scheurer Christian Hinderling}

Zurich University of Applied Sciences, Institute of Chemistry and Biological Chemistry Postfach, CH-8820 Wädenswil, Switzerland

Reversible adhesion is required in many different applications. Well known approaches for solving this problem are hook-and-loop fasteners (Velcro ${ }^{\circledR}$ ) as well as sticky notes (post-it $\left.{ }^{\circledR}\right)$. Nevertheless, these solutions are not suitable for certain applications, e.g. for textile applications where the rough feel of Velcro hooks is highly uncomfortable.

Electrically charged polymer fibers are already used for filtration, as the electrostatic interaction improves the particle capture mechanism [1]. Here we report a novel approach to obtain reversible adhesion by producing oppositely charged polymer thin films.

The polymer thin films with a defined charge were produced with layer-bylayer deposition of polyelectrolytes [2] by means of dip-coating. The surface charge of the polymer films was analyzed by electrostatic force Microscopy (EFM) which allows mapping the electrical properties at the nanoscale.

First adhesion experiments have indeed shown evidence that reversible interaction between oppositely charged surfaces can be achieved.

[1] J. Kim, W. Jasper, J. Hinestroza, J Mircrosc. 2007, 225, 72.

[2] G. Decher, Science, 1997, 277, 1232 
Polymers \& Colloids

Metal-NTA-functionalized vesicles for molecular recognition

\author{
Pascal Tanner $^{1}$, Rainer Nehring ${ }^{1}$, Cornelia G. Palivan ${ }^{1}$, Wolfgang Meier ${ }^{1}$
}

${ }^{1}$ University of Basel/Department of Chemistry, Klingelbergstrasse 80, CH4056 Basel, Switzerland

Molecular recognition at surfaces plays a key role in many processes in nature. Recently various copolymer membranes were modified for molecular recognition by attaching specific sites, such as biotin or integral membrane protein receptors.[1][2] We developed a new approach by introducing metal-nitrilotriacetic acid (metal-NTA) functionalized amphiphilic diblock copolymers, which self-assemble in nanovesicles with the metal exposed at their outer surface, able to specifically bind to his-tagged proteins.[3]

Here we present how we developed the metal-NTA functionalized poly(butadiene)-block-poly(ethylene oxide) (PB-b-PEO) vesicles in various environmental conditions in order to maximize the protein binding affinity. We check the ability of the metal-functionalised polymeric vesicles to selectively bind to oligohistidine residues $\left(\mathrm{His}_{6}-\mathrm{Tag}\right.$ ) of proteins.

Vesicles formation was investigated by LS, SAXS, TEM, and zeta-potential measurements. Protein attachment was visualized by fluorescence microscopy and quantified with fluorescence correlation spectroscopy (FCS) and electron paramagnetic resonance spectroscopy. The dissociation constants determined by FCS indicate a significantly improved binding in an optimised hybrid system based on a mixture of metal-functionalised/nonfunctionalised copolymers with $15 \% \mathrm{Ni}$ ions. The system was very sensitive in solution at $\mathrm{pH} 7.4$ for all model his-residues and his-tagged proteins, which supports further applications based on molecular recognition.

[1] Rigler, P., Meier, W. JACS 2006, 128, 367-373.

[2] Graff, A.; Sauer, M.; Van Gelder, P.; Meier, W. PNAS 2002, 99, 5064-5068.

[3] Nehring, R.; Palivan, G.C.; Casse, O.; Tanner, P.; Tuxen J.; Meier W. Langmuir, 2008, 25(2), 1122-1130.

Polymers \& Colloids

Synthesis of Poly(m-phenylene)s and Poly( $m, p$-phenylene)s by Suzuki Polycondensation

Benjamin Hohl, Samuel Jakob, Junji Sakamoto*, A. Dieter Schlüter*

ETH Zurich, Laboratory of Polymer Chemistry, Department of Materials, Wolfgang-Pauli-Strasse 10, CH-8093 Zürich, Switzerland

Polyphenylenes attract increasing attention due to their unique mechanical and optoelectronic properties. Suzuki Polycondensation (SPC) is often used both in academia and in industry and recognized as the most robust method for the synthesis of this class of polymers [1]. In the present study, SPC of $m$-phenylene monomers with various side chains leading to $\operatorname{poly}(m-$ phenylene)s and poly $(m, p$-phenylene)s is investigated. These SPCs intrinsically afford not only linear polymers but also cyclic products. The SPC conditions have been optimized in order to achieve high molar mass polyphenylenes aiming at novel polymeric materials [2].

(a)<smiles>[R]c1cc(Br)cc(B2OC(C)(C)C(C)(C)O2)c1</smiles>

(b)

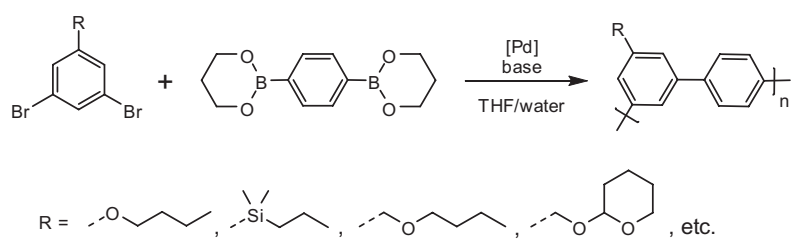

[1] J. Sakamoto, M. Rehahn, G. Wegner, A. D. Schlüter, Macromol. Rapid Commun., 2009, 30, 653.

[2] R. Kandre, K. Feldman, H. E. H. Meijer, P. Smith, A. D. Schlüter, Angew. Chem. Int. Ed. 2007, 46, 4956.
Polymers \& Colloids

Optimization of the Efficiency of Flame Retardant Polymers on Cotton with a Triacin-Coupling-Agent

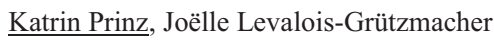

Department of Chemistry and Applied Biosciences, ETH Zürich 8093-Zürich, Switzerland

Certain phosphorous compounds are known to alter the thermal decomposition process of treated polymers. It has been shown, that diethyl acryloyloxy ethyl phosphoramidate is a qualified flame retardant for cotton. [1] This flame retardant is polymerized on the surfaces of textiles protecting them from the outside by cold plasma technology. However, at high enough oxygen contents the cellulose fibres are burning from the inside. Increased protection efficiency of the polymer-films can be achieved by reducing the decomposition of the cellulose from the inside.

To this end a new dichlortriacin-anchor linked to an acrylic group as polymerizable functionality was synthesized. This crosslinker was applied to cotton textile like a reactive dye by a cold pad batch process. The pretreated cotton was then finished with phosphorous compounds as flame retardants using the cold plasma technology.

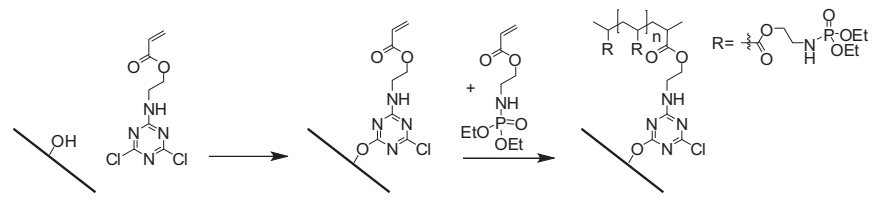

Cellulose treated with the new crosslinker has a superior Limited Oxygen Index (LOI) compared to a non pre-treated textile. The crosslinking between fibres and the covering flame retardant helps to prevent the burning process.

[1] M. J. Tsafack, J. Levalois-Grützmacher, Surf. Coat. Technol. 2006, 201, 2599-2610.

Polymers \& Colloids

Towards 2D Coordination Polymers Using Shape-Persistent Monomers with $\boldsymbol{m}$-Terpyridinyl Units at the Periphery

Thomas Bauer, A. Dieter Schlüter,* Junji Sakamoto*

ETH Zurich, Laboratory of Polymer Chemistry, Department of Materials, Wolfgang-Pauli-Strasse 10, CH-8093 Zurich, Switzerland

A laterally infinite, one monomer-unit thick, free-standing unimolecular network with a defined internal structure is referred to as $2 \mathrm{D}$ polymer [1]. There have been many attempts to synthesize such polymers but none of them met with success so far. In the present study, a set of $D_{3 \mathrm{~h}}$ and $D_{6 \mathrm{~h}}$ symmetric shape-persistent monomers with $m$-terpyridinyl units were synthesized. These monomers are expected to grow under thermodynamic control through reversible complexation with metal cations such as $\mathrm{Zn}^{2+}, \mathrm{Co}^{2+}$ and $\mathrm{Ru}^{2+}$. The obtained polymer structures were analyzed by AFM, TEM, STM as well as UV/Vis absorption and fluorescence spectroscopies, etc.

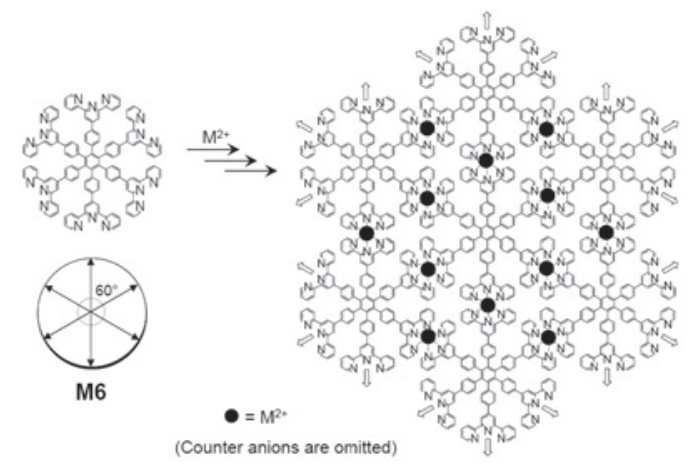

[1] J. Sakamoto, J. van Heijst, O. Lukin, A. D. Schlüter, Angew. Chem. Int. Ed. 2009, 48, 1030

[2] T. Bauer, A. D. Schlüter, J. Sakamoto, Synlett 2010, 877. 
Application of Bis(Acyl)phosphane Oxides in Photo Emulsion Polymerization of Small Polymer Beads of Narrow Dispersity

\author{
Judith Bräuer, Hansjörg Grützmacher
}

Department of Chemistry and Applied Biosciences, ETH Zürich, WolfgangPauli-Str. 10, 8093 Zürich, Switzerland

Photoinitators (PI) are of great importance for several processes, e. g. in the photo-induced curing in dentistry, coating or printing technologies [1]. One important class of PI's is bis(acylphosphane) oxides (BAPO) which initiate reactions by radiation with UV or visible light.

New oligo(ethylenglycol) functionalized BAPOs were synthesized to increase the water solubility in order to apply them in emulsion polymerization processes. Photolysis of these BAPOs in the presence of an emulsion of styrene, sodium dodecyl sulfate and water yielded almost mono-disperse polymer beads with sizes below $50 \mathrm{~nm}$ in high yields. The polymers were characterized by Scanning Electron Microscopy (SEM), Dynamic Light Scattering (DLS) and Size Exclusion Chromatography (SEC).

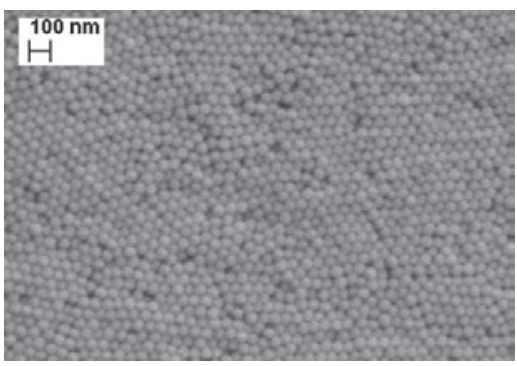

[1] K. Dietliker, Photoinitiators for Free Radical Cation. and Anion. Photopolymerization, $2^{\text {nd }}$ Edition 1998, SITA Technology Ltd. London.

Polymers \& Colloids

Diffusion of Small Molecules in Polymers:

\section{a Lattice Free-Volume Theory}

\section{I. Costa, G. Storti, M. Morbidelli}

ETH Zürich, Institute for Chemical and Bioengineering HCI-F138, Wolfgang-Pauli-Str. 10, 8093 Switzerland

The rate of transport of small molecules in polymers, such as monomers, oligomers, plasticizers or additives plays a key role in both polymer reaction, polymer processing and in applications where sorption/desorption kinetics are involved (e.g. drug release from polymer matrixes). Despite that, and the numerous models proposed in past years, to date no fully predictive theory exists to evaluate diffusion coefficients in polymers. ${ }^{1}$

Here we show that the Free Volume (FV) concept may be used as a bridge between kinetic and thermodynamic theories: assuming a correspondance between the FV and the unoccupied volume of pure components as given by Lattice Theories (e.g. the Sanchez-Lacombe molecular theory ${ }^{2}$ ) a new equation is obtained for the calculation of self diffusion coefficients in multicomponent mixtures. ${ }^{3}$ A parameter evaluation scheme is proposed, which is substantially simpler compared with the conventional Vrentas-Duda approach, ${ }^{4}$ even without losing generality. The theory is compared with experimental data of self diffusion coefficients for several solvent/polymer binary mixtures above the mixture glass transition temperature in wide ranges of physical conditions showing that good correlative ability is achieved in all cases.

[1] L. Masaro, X. X. Zhu, Prog. Polym. Sci. 1999, 24, 731.

[2] I. C. Sanchez, R. H. Lacombe, Macromolecules 1978, 11, 1145.

[3] L. I. Costa, G. Storti, J. Polym. Sci. Part B: Polym. Phys. 2010, 48, 529.

[4] J. S. Vrentas, J. L. Duda, J. Polym. Sci. Polym. Phys. Ed. 1977, 15, 403 .

\section{Controlled Growth of Protein Dendrimers}

Sabina Burazerovic, Thomas R. Ward*

University of Basel/ Inorganic Chemistry department, Spitalstr. 51, CH4056 Basel.
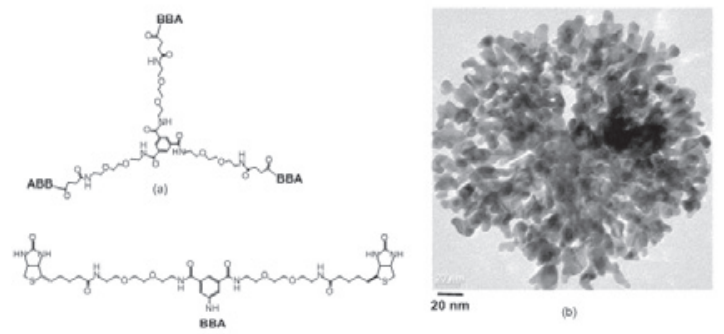

Figure 1 Bis-biotinylated building blocks for the hierarchical protein assembly. The trifurcated ligand (a) affords dendrimer-like polymer (b) when reacted with streptavidin.

Over the last decades, tremendous efforts have been invested worldwide to gain more insight into self-assembly processes in order to produce new materials through the so-called bottom-up approach. On the basis of the work which has been previously reported in our lab [1], we are now trying to reach higher orders of self-assembled protein nanostructures. Introduction of a trifurcated bis-biotinylated anchor (BBA) in the presence of streptavidin produces dendrimer like structures (Fig. 1). In order to obtain and characterize monodisperse polymeric material, we immobilize the protein networks via biotinylated SAMs on gold surfaces. Sequential addition of streptavidin followed by the BBA linkers allows us to create well defined dendrimeric structures of increasing size. AFM, SPR and QCM are currently used to characterize these protein polymers

[1] Sabina Burazerovic, Julieta Gradinaru, Julien Pierron, Thomas R. Ward, Angew. Chem. Int. Ed. 2007, 46, 5510-5514.

Polymers \& Colloids

\section{Nanostructures from Oligopeptide-Polymer Conjugates}

\author{
Liangfei Tian ${ }^{1,2}$, Ruth Szilluweit ${ }^{1}$, Holger Frauenrath ${ }^{1 *}$
}

${ }^{1}$ EPFL/Laboratory of Macromolecular and Organic Materials, Institute of Materials, EPFL - STI - IMX - LMOM, Building MXG, Room 037, Station 12,1015 Lausanne,Email: holger.frauenrath@epfl.ch ${ }^{2}$ ETH Zürich/Institute for Polymer Chemistry, ETH Hönggerberg, Wolfgang-Pauli-Strasse 10, 8093 Zürich

Supramolecular self-assembly has proven to be a versatile tool for the preparation of nanostructured materials. ${ }^{1}$ Various examples utilize the selfassembly of $\beta$-sheet forming oligopeptides. Recently, we have demonstrated that oligopeptide-polymer conjugates based on hydrogenated poly(isoprene) and oligo(alanine) can be used for the formation of soluble nanostructures in a "bottom-up" approach. ${ }^{2}$ In the present study, we prepared and investigated a series of diacetylene macromonomers which comprised hydrogenated poly(isoprene) segments with different degrees of polymerization as well as oligo(alanine)s of different lengths. The resulting nanostructures were visualized by AFM, and their stability as well as polymerizability were investigated by solution phase IR and UV-vis spectra. Our investigations showed that the stability of the nanostructures in solution and their polymerizability was strictly determined by both the length of the attached polymers and the number of $\mathrm{N}-\mathrm{H} \cdots \mathrm{O}=\mathrm{C}$ hydrogen bonds.

The lessons learned from the diacetylene conjungates were then transferred to oligopeptide-polymer-equipped derivatives of other conjungated molecules such as tetra(thiophene)s. A systematic investigation of UV-vis, CD, and IR spectra as well as AFM images revealed that oligopeptide-polymer conjugates based on hydrogenated poly(isoprene) and oligo(alanine) were versatile scaffolds for reproducible formation of one-dimensional aggregates of conjugated molecules.

[1] Lehn, J.-M. Science 2002, 295, 2400.

[2] Jahnke, E.; Weiss, J.; Neuhaus, S.; Hoheisel, T. N.; Frauenrath, H. Chem. Eur. J. 2009, 15, 388. 
Polymers \& Colloids

Single-Chain Nanoparticles by Collapse of Waters Soluble Polymers

\section{Bandera, E. W. Meijer*}

Institute for Complex Molecular Systems and Laboratory of Macromolecular and Organic Chemistry, Eindhoven University of Technology, P. O. Box 513, 5600 MB Eindhoven, The Netherlands

E-mail: e.w.meijer@tue.nl

In nature, the preparation of well-defined macromolecules from a relatively small number of building blocks is highly efficient leading to structurally organized and functional architectures. Chemical synthesis provides a vast amount of polymeric materials but, the understanding and the tuning of their three dimensional arrangement is still challenging. Recently, the preparation of single-chain polymeric nanoparticles by irradiation-induced collapse of a polymer with dangling and protected hydrogen bonding cross-linking groups (2-ureido-pyrimidinone, UPy), was reported..$^{[1],[2]}$ The aim of this project is the preparation of water-soluble polymers containing protected UPy groups and the study of their folding to nanometer sized objects upon irradiation with UV-light. The materials of choice are polynorbornene succinimides, details on the structure and physical properties of the particles will be addressed.
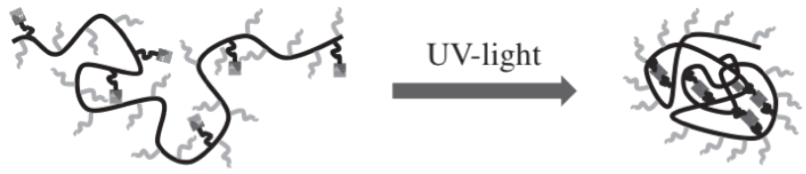

= UPy-protected cross linking groups

[1] E. J. Foster, E. B. Berda, E. W. Meijer, J. Am. Chem. Soc. 2009, 131, 6964.

[2] E. B. Berda, E. J. Foster, E. W. Meijer, Macromolecules 2010, 43, 1430 .

Polymers \& Colloids

Understanding the breakage process of open \& dense colloidal aggregates in contracting nozzle

Yogesh M. Harshe, Miroslav Soos, Marco Lattuada, Massimo, Morbidelli

Institute for Chemical and Bioengineering, Department of Chemistry and Applied Biosciences, ETH Zurich, 8093 Zurich, Switzerland

Colloidal suspensions are usually exposed to sheared flow fields for their processing, size modification (aggregation or breakage), and separation. The dynamic evolution of morphologies and/or size and size distribution of the aggregates in the suspension are strongly dependent on their initial conditions. Experiments in this direction always help in quantifying the dependence of aggregate structure and size on the applied flow ${ }^{1}$, however, lack detailed understanding of the microscopic flow and force balances. On the other hand, modeling efforts are useless unless supported by some experimental evidences.

We have studied the breakage process of two types of diffusion limited colloidal aggregates (DLCA) namely, static DLCA $\left(d_{f}=1.7\right)$ and flow-induced DLCA $\left(d_{f}=2.7\right)$, generated from $90 \mathrm{~nm}$ and $810 \mathrm{~nm}$ primary particles. The aggregates were broken in elongational flow generated in a contracting nozzle. The evolution of cluster mass and morphology was tracked by light scattering measurements and laser microscopy, wherever possible. The experimental results were compared with Stokesian dynamics ${ }^{2}$ model incorporating the Van der Waals' interactions, Born repulsion forces, and also the tangential interactions as described by Becker and Briesen ${ }^{3}$. The simulation results were compared with the experimental findings for qualitative evolution of aggregates' mass and morphology, which helped in decoupling the breakage and restructuring phenomena.

[1] Soos, M., Ehrl, L., Babler, M. U. and Morbidelli, M., Langmuir, 2010, 26,10

[2] Brady J. F. and Bossis G., Ann. Rev. FluidMech., 1988, 20, 111

[3] Becker V. and Briesen H., Phys. Rev. E, 2008, 78(6), article 061404
Polymers \& Colloids

Blends of semiconducting small molecules and insulating polymers for next-generation organic based opto-electronic devices

$\underline{\text { Pascal Wolfer }}^{1}$, Assunta Marrocchi ${ }^{2}$, Maria Laura Santarelli ${ }^{3}$, Alessandra Broggi ${ }^{3}$, Natalie Stingelin ${ }^{1,4}$, Paul Smith ${ }^{1}$

${ }^{1}$ Department of Materials, ETH Zürich, CH-8093 Zürich, Switzerland

${ }^{2}$ University of Perugia, Department of Chemistry, I-06123 Perugia, Italy

${ }^{3}$ University of Rome La Sapienza, DICMA, I-00185 Roma, Italy

${ }^{4}$ Department of Materials, Imperial College London, London SW7 2AZ, UK

Organic semiconductors based on $\pi$-conjugated small molecules and polymers are widely studied as the active layer in a variety of (opto-)electronic applications. Currently, an increasing interest is directed towards semiconductor:insulator blend systems that comprise a (semi-)crystalline polymer as the matrix material, providing significantly enhanced stability and mechanical robustness to the binary. Under judiciously chosen processing conditions, this approach allows preparation of continuous thin films with excellent electronic performance at remarkably low semiconductor contents as measured in field-effect transistors (OFETs) and photovoltaic cells (OPVs) $[1,2]$. Hence, in this study we demonstrate that detailed understanding of the phase behavior of multifunctional arylacetylene:polymer binaries allows controlling and tailoring of the blend microstructures induced and, thus, optimization of the electronic device performance as presented for OFETs [3].

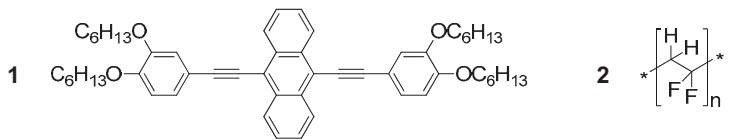

Figure 1: Chemical structures of blend components employed: (1) 9,10-bis$\{[m, p$-bis(hexyloxy)phenyl] ethynyl $\}$ anthracene and (2) poly(vinylidene fluoride) (PVDF).

[1] P. Wolfer et al., Synth. Met. 2007, 157, 827.

[2] S. Goffri et al., Nature Mater. 2006, 5, 950.

[3] A. Marrocchi et al., Chem. Commun. 2009, 1380.

Polymers \& Colloids

Hydrodynamic Properties of fractal aggregates

Yogesh M. Harshe, Marco Lattuada $^{1}$

${ }^{1}$ Institute for Chemical and Bioengineering, Department of Chemistry and Applied Biosciences, ETH Zurich, 8093 Zurich, Switzerland

The hydrodynamic properties of rigid fractal aggregates are key ingredients in understanding the governing mechanism of their motion and the properties of their suspensions [1]. In the present work we outline explicit equations for the estimation of the complete set of hydrodynamic properties of arbitrary shaped aggregates made of uniform sized spherical primary particles. The rigid body motion equations are coupled to Stokesian dynamics [2] model to derive rigid body properties. A huge library of clusters consisting of fractal aggregates of different morphologies $\left(d_{f}=1.8\right.$ to 3.0$)$ and spheroids of different axes ratios with wide range of number of constitutive spheres was used. Using the developed rigid body equations the average cluster grand resistance matrix has been computed and its relationship with cluster mass and morphology has been investigated. In the effort to introduce a simplified approach that accurately reproduces the complex hydrodynamic behavior of an arbitrary fractal cluster, including its anisotropic behavior, an equivalent ellipsoid model has been introduced. A cluster is represented by an ellipsoid having the same principal moments of inertia as the cluster. The predicted hydrodynamic properties of the equivalent ellipsoid, which can be computed exactly, closely match those of the clusters, for all cluster masses and morphologies. Therefore, this simplified approach provides an effective tool to predict the behavior of any cluster with complex structure.

[1] H. Brenner and D. W. Condiff, J. Colloid. Interface. Sci., 1972, 41

(2), 228

[2] Brady J. F. and Bossis G., Ann. Rev. FluidMech., 1988, 20, 111 


\section{Kinetic Analysis of Ring Opening Polymerization of L,L-Lactide}

Yingchuan Yu, Giuseppe Storti, Massimo Morbidelli ${ }^{*}$

ETH Zurich, Institute for Chemical \& Bioengineering, Wolfgang-Pauli Str. 10, CH-8093 Zurich, Switzerland

As one of the most well-known degradable polymers, poly(lactic acid) (PLA) is considered as the most promising substituent of petroleum-based polymers for daily use. To properly scale up the production of PLA from lab flask to large scale industrial plant, reliable mathematic models are indeed valuable tools.

Aiming to fill the gap in PLA modeling study, a systematic experimental and modeling work on ring opening polymerization of L,L-Lactide in bulk using the FDA approved $\mathrm{Sn}(\mathrm{Oct})_{2}$ as catalyst and 1-dodecanol as co-catalyst has been carried out. A wide range of reaction temperatures have been examined. Based on the alkoxide initiation mechanism [1], the model exhibits remarkably good fitting to the experimental data. The rate coefficients of all involved reactions have been evaluated at different temperatures and for a large set of recipe conditions. Major achievements from this analysis are: (i) the development of a model is suitable to calculate the average properties of the polymer molecular weight, (ii) the evaluation of the equilibrium constant of catalyst activation, the key parameter when designing the reaction recipe, (iii) the understanding of the interplay between the transfer reactions involving the alcoholic co-catalyst and the transesterification reactions [2]. Finally, using the developed kinetic model along with the corresponding parameter values, the performance of different combinations of ideal reactors can be examined in order to identify most promising process conditions. This part of the work is going on in collaboration with the company Uhde-Inventa Fischer (Germany) aimed to design industrial scale PLA production facilities [3]

[1] Duda, A., Penczek, S, In Biopolymers, Volume 3b, Doi, Y., Steinbüchel, A., Ed. Wiley-VCH, Weinheim: 2002; pp 371.

[2] Yu, Y. C., Storti, G., Morbidelli, M., Macromolecules, 2009, 21, 8187.

[3] KTI Project No.: 8611.2 PFIW-IW.

Polymers \& Colloids

580

Study of the influence of the hydrotalcite- $\mathrm{CO}_{3}$ addition to hybrid sol-gel films.

R.Losada, A. Collazo, X.R. Nóvoa, C. Pérez

Corrosion and Materials Engineering Group (ENCOMAT), ETSEI, University of Vigo, Campus Universitario, 36310 Vigo (Spain)

The toxicity of classical pretreatments based on chromate conversion coatings (CCCs) makes necessary the development of new healthy and environmentally safe alternatives. Sol-Gel coatings are a good alternative; they are environmentally friendly and provide good adhesion not only with metallic substrate but with the subsequent organic coatings. The sol-gel films consist in an organic-inorganic network obtained by hydrolysis and condensation reactions from metallic alkoxides. In addition, the addition of particles with a potential inhibition effect may enhance the anticorrosive performance. Hydrotalcites are being studied like an environmental friendly alternative. They are good anionic exchangers, except when the compensating ion is $\mathrm{CO}_{3}{ }^{2-1}$. Nevertheless previous studies performed by our research group have shown a corrosion resistance improvement when hydrotalcite$\mathrm{CO}_{3}{ }^{2-}$ is added to the sol-gel ${ }^{2}$. The present work is focus on the curing kinetics study of the sol-gel film with and without HT particles, and in elucidating the possible reaction mechanism of hydrotalcites- $\mathrm{CO}_{3}{ }^{2-}$.

[1] Miyata S. Clays and clay minerals, 1983, 31,4, 305.

[2] Alvarez D, Collazo A, Hernández M, Nóvoa XR, Pérez C. Prog. Org. Coat., 2010, 67,2,152
Novel Biodegradable Nanoparticles and Their Applications in Drug Delivery

Yingchuan Yu, Davide Moscatelli, Giuseppe Storti, Massimo Morbidelli*

ETH Zurich, Institute for Chemical \& Bioengineering, Wolfgang-Pauli Str. 10, CH-8093 Zurich, Switzerland

Poly(lactic acid) (PLA) nanoparticles are considered effective devices for drug delivery and targeting, mainly because of their degradable/biocompatible nature of the polymer along with the suitable particle size. There are lots of methods to prepare PLA particles of colloidal size, but the minimum particle size produced through any technique is around $100 \mathrm{~nm}$.

The aim of this work is to assess a new approach to the synthesis of PLA particles with smaller size. Through ring-opening polymerization of L,L-Lactide using FDA approved $\mathrm{Sn}(\mathrm{Oct})_{2}$ as catalyst and suitable co-catalysts, functionalized PLA are produced. Starting from these PLA and applying conventional emulsion polymerization process, extremely small PLA-based, fully degradable nanoparticles with size down to $17 \mathrm{~nm}$ are synthesized. Meanwhile, extremely narrow particle distributions have been obtained. The degradation study has been carried out using different particles in terms of size and PLA chain length as well. The rates of degradation and release of lactic acid and its oligomers from the nanoparticles have been measured by high performance liquid chromatography (HPLC). The complete degradation kinetics of the PLA-block of the polymer chains has been studied. Finally, the biocompatibility of these particles has been proved by in vitro tests. The nanoparticles stabilized by TWEEN 80 have been incubated with U266 myeloma cell line at $37^{\circ} \mathrm{C}$. The time-increasing viable cell counts and the high viability of the cells at the end prove non-toxicity

Such ultra-small biodegradable nanoparticles are very promising for special drug delivery applications. In particular they can be used to enhance the cellular uptake and to increase the membrane passage, especially the blood brain barrier (BBB). Similar studies are currently on going in collaboration with the Institute for Pharmacological Research Mario Negri (Milano, Italy).

\section{Polymers \& Colloids}

New generation of crash-durable structural adhesives: excellent adhesion, good temperature stability and a long shelf life

\section{Christof Brändli, Andreas Lutz}

Dow Europe GmbH, Bachtobelstrasse 3, 8810 Horgen, Switzerland

For more than 10 years, crash-resistant adhesives have been used to bond components of vehicle body shells. These products are responsible, among other things, for lending additional crash resistance, stiffness and fatigue strength to the bodywork components, which are bonded and spot-welded together. A new generation of these adhesives, with properties that have been further improved, has been developed and is about to be launched on the market. The high viscosity, first generation products were pumped and applied using heated machines. The second generation adhesives could also be sprayed onto surfaces and were characterised by high strength and resistance to moisture. This enabled the application speed to be increased significantly and the spray nozzle and the component to be positioned much further apart. As a result, crash-resistant adhesives could be applied relatively easily to complex components. At the same time, the problem of washout was resolved by the use of specially developed polymers.

By developing specific polymers it has now been possible to increase significantly the temperature stability of crash-resistant structural adhesives during storage and processing. At the same time, bonding agents have been incorporated into the polymers with the aim of improving the adhesion of the products to metal surfaces during the application process and after curing, in order to reduce the risk of corrosion. The adhesives are less viscous and have a higher yield strength than previous products. In addition, they are easier to apply and can bridge wider gaps. This presentation describes the benefits for the application process of the improvements to:

- the thermal stability of the adhesives

- the adhesive rheology

- and the adhesion properties. 